\title{
UV-to-IR Absorption of Molecularly p-Doped Polythiophenes with Alkyl and Oligoether Side Chains: Experiment and Interpretation Based on Density Functional Theory
}

\author{
Ihor Sahalianov, Jonna Hynynen, Stephen Barlow, Seth R. Marder, Christian Müller, \\ and Igor Zozoulenko*
}

Cite This: J. Phys. Chem. B 2020, 124, 11280-11293

Read Online

\section{ACCESS | Lill Metrics \& More | 期 Article Recommendations | (1) Supporting Information}

ABSTRACT: The UV-to-IR transitions in p-doped poly(3-hexylthiophene) (P3HT) with alkyl side chains and polar polythiophene with tetraethylene glycol side chains are studied experimentally by means of the absorption spectroscopy and computationally using density functional theory (DFT) and tight-binding DFT. The evolution of electronic structure is calculated as the doping level is varied, while the roles of dopant ions, chain twisting, and $\pi-\pi$ stacking are also considered, each of these having the effect of broadening the absorption peaks while not significantly changing their positions. The calculated spectra are found to be in good agreement with experimental spectra obtained for the polymers doped with a molybdenum dithiolene complex. As in other DFT studies of doped conjugated polymers, the electronic structure and assignment of optical transitions that emerge are qualitatively different from those obtained through earlier "traditional" approaches. In particular, the two prominent bands seen for the p-doped materials are present for both polarons

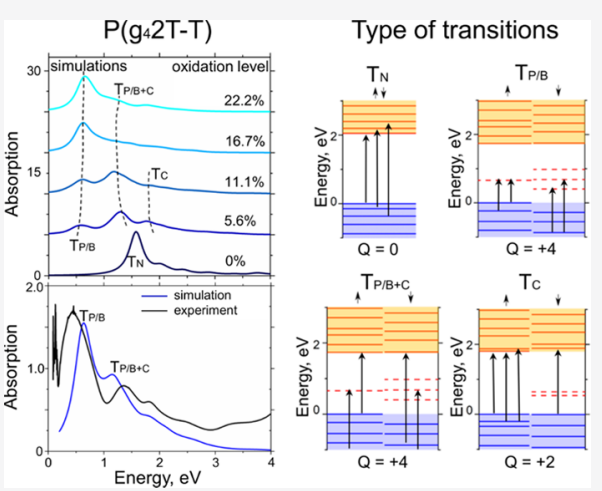
and bipolarons/polaron pairs. The lowest energy of these transitions is due to excitation from the valence band to a spin-resolved orbitals located in the gap between the bands. The higher-energy band is a superposition of excitation from the valence band to a spin-resolved orbitals in the gap and an excitation between bands.

\section{INTRODUCTION}

Molecular doping and side-chain engineering of conducting polymers represent powerful tools to enhance and tune the electronic and optical properties of polymer-based devices. Doping leads to electron transfer between dopants and the polymer backbone, thus increasing the charge carrier concentration. This, in turn, can strongly affect the mobility and the polymer morphology due to changes in the mechanical stiffness/planarity of backbones and the enhancement of backbone-dopant interactions. ${ }^{1-4}$ The utilization of oligoether side chains greatly improves polymer solubility in most organic solvents. ${ }^{5}$ Because of their hydrophilicity, oligoether side chains enhance the intake of hydrated ions and water swelling. ${ }^{6-8}$ The interplay between molecular doping and the character of the side chains makes it possible in some cases to alter morphological and electrical properties of polymer thin films, enhancing their crystalline order, thermal stability, conductivity, Seebeck coefficients, and other important material parameters. These changes, can in turn, lead to improved performance of polymer-based devices such as organic solar cells, ${ }^{9}$ field-effect transistors, ${ }^{10}$ electrochemical transistors, ${ }^{11}$ actuators, and robotic devices. ${ }^{12}$

Most of the conducting polymers are $\mathrm{p}$-doped because the Fermi level in the electrodes on the metal/polymer interface is typically aligned with the valence band of polymer, which favors the hole injection. ${ }^{13}$ Molecular doping of polythiophenes (PTs) and related polymers has been a subject of intensive research (see, e.g., refs 13 and 14 for a review). Molecular p-doping of thiophene-based polymers with different side chains such as poly(3-hexylthiophene) (P3HT), with aliphatic side chains, ${ }^{14,15}$ and poly $\left(13,13^{\prime}-\left(\left[2,2^{\prime}: 5^{\prime}, 2^{\prime \prime}\right.\right.\right.$-terthiophene]-3,3'-diylbis( oxy)) bis (2,5,8,11-tetraoxatridecane)) P$\left(\mathrm{g}_{4} 2 \mathrm{~T}-\mathrm{T}\right)^{15,16}$ with tetraethylene glycol side chains have been studied (see Figure 1 for chemical structure). The studies referred to above focused on electronic properties including conductivity and Seebeck coefficient. Also, the optical spectra of these materials were reported. It should be noted, however, that a detailed theoretical understanding of the effect of doping and side chains on the optical absorption by these polymers is not complete.

Optical absorption spectroscopy of polythiophenes and related conjugated polymers with a thiophene-like backbone

Received: September 25, 2020

Revised: November 4, 2020

Published: November 25, 2020 


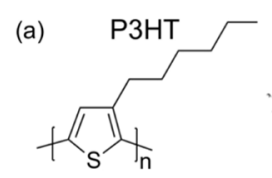

(b) $\quad \mathrm{P}\left(\mathrm{g}_{4} 2 \mathrm{~T}-\mathrm{T}\right)$

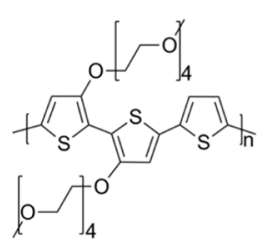

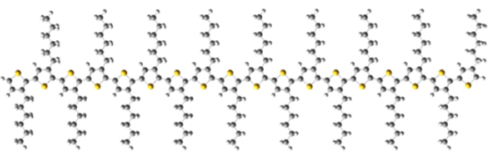

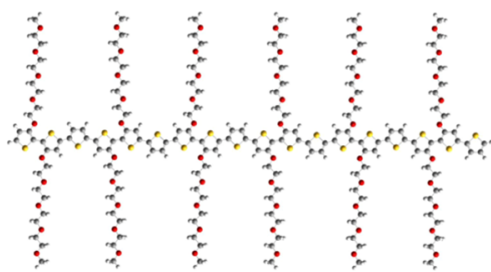

(c) $\mathrm{Mo}\left(\mathrm{tfd}-\mathrm{COCF}_{3}\right)_{3}$

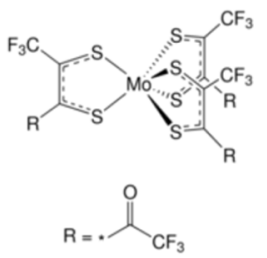

Figure 1. Chemical structure of (a) P3HT, (b) $\mathrm{P}\left(\mathrm{g}_{4} 2 \mathrm{~T}-\mathrm{T}\right)$, and (c) $\mathrm{Mo}\left(\mathrm{tfd}-\mathrm{COCF}_{3}\right)_{3}$. Polymers are visualized with two oligomers consisting of $N$ $=18$ thiophene units each.

such as poly(3,4-ethylenedioxythiophene) (PEDOT) has received tremendous attention during past decades. ${ }^{16-26}$ When the oxidation level of the polymer is varied, the optical absorption spectra evolve and show features that are similar for all the above-mentioned polymers. Namely, undoped polymer films show absorption at $\approx 2.0-2.3 \mathrm{eV}(450-600 \mathrm{~nm})$ due to the electronic transition from the valence to the conduction band. The absorption peaks of the neat polymer gradually disappear as the doping level increases. At the same time, a peak at intermediate energies $\approx 1.3-1.7 \mathrm{eV}(700-900 \mathrm{~nm})$ accompanied by a peak at low energies $\approx 0.3-0.7 \mathrm{eV}(>1500$ $\mathrm{nm}$ ) develop. As the oxidation level increases further, the absorbance of the intermediate-energy peak reaches a maximum and then decreases, while that of the low-energy peak increases and becomes dominant in the spectra.

In the literature, one can find various interpretations of the nature of these peaks in the electronic absorption spectra and the explanation of their evolution during oxidation and reduction of thin films. An early interpretation, which is still often invoked in the current literature, was developed in the 1980s and was based on various methods, including SuSchrieffer-Heeger Hamiltonian, ${ }^{27}$ valence effective Hamiltonian, ${ }^{28}$ various Hartree-Fock methods, ${ }^{17,29,30}$ Holstein Hamiltonian, ${ }^{31}$ and others. Following Heimel, ${ }^{32}$ we will refer to these approaches and corresponding interpretations as "traditional” ones. In recent years, density functional theory (DFT) and time-dependent (TD)-DFT methods have been widely utilized to study various aspects of electronic structure and optical absorption in polythiophenes and related polymers. $^{22,32-44}$ These methods have also been applied to $n$ doped polymers ${ }^{32,45}$ and p-type/n-type blends. ${ }^{46}$ It has been realized that DFT-based approaches lead to a qualitatively different electronic structure and provide a distinct interpretation of the absorption peaks and the evolution of the spectra as compared to the above-mentioned traditional picture (see Section III.I for discussion of the differences in the traditional and DFT descriptions). ${ }^{22,32,36,41}$ The essential reason for these differences is that the traditional model fails to capture some very basic and essential physics; specifically, it ignores the Coulomb interactions leading to the lifting of the spin degeneracy that DFT calculations take into account effectively. These effects have been discussed more recently in papers by Png et al., ${ }^{43}$ Winkler et al., ${ }^{47}$ and Heimel $^{32}$ in a very accessible manner, based upon a combination of experimental studies aided by DFT studies.

In spite of the qualitatively and quantitatively different picture that modern DFT-based approaches provide (see
Section III.I), they remain largely ignored by many experimental studies on polythiophenes and related polymers. The current literature, ${ }^{19,20,24-26}$ including many recent reviews, ${ }^{48-51}$ is still dominated by the early traditional picture. Hence, we argue that DFT studies that elucidate the electronic structure of conjugated polymers are vital to provide more accurate descriptions of these transitions, despite the limitation of various levels of DFT (for example, the tendency to overdelocalize electrons).

As briefly reviewed above, many DFT-based studies of polythiophenes and related polymers addressing various aspects of the electronic structure and the nature of charge carriers have been reported during past decades. However, a number of crucial questions remain unanswered. One such question is the effect of doping on the electronic structure and optical transitions. Most DFT-based studies focusing on the absorption spectra for the systems at hand consider only the isolated charged oligomers and neglect the influence of compensating charge associated with the anions that results from the dopants and which modifies the electrostatic potential around the positively charged $\pi$-systems. Providing such an understanding of their effect on the electronic structure and corresponding optical transitions represents one of the central aims of this study.

Other questions that are not clarified yet within the DFT approach concern the effect of a realistic nanostructure on the electronic absorption spectra. In particular, polythiophenes tend to form $\pi-\pi$-stacked aggregates and in some cases crystallites composed of several chain segments. ${ }^{25,31,44,48,52}$ Therefore, the effect of $\pi-\pi$ stacking on the absorption spectra needs to be quantified. Further, at finite temperatures, the chain segments and crystallites that constitute the solid-state nanostructure are in constant movement, leading to twisting of main-chain bonds and changes in torsion angles and movements of the side chains. While a standard DFT calculation is suitable for accurate simulations of individual polymer chains, it is too computationally expensive to simulate $\pi-\pi$ stacks when one needs to average over many dopant configurations or temperature-induced motion of oligomers with long side chains. In the present paper, we utilize a molecular dynamics (MD) simulation based on tight-binding DFT (DFTB) and provide a systematic treatment of the above-mentioned effects.

Here, we report on a combined experimental and theoretical study of absorption spectroscopy of doped P3HT and P $\left(\mathrm{g}_{4} 2 \mathrm{~T}\right.$ $\mathrm{T}$ ) and interpret the observed spectra based on the electronic structure and corresponding electronic transitions calculated 
using DFT and molecular dynamics simulations utilizing tightbinding DFT. Through the utilization of the DFT-based approaches, we are able to systematically study and quantify the influence of the above-mentioned key factors on the absorption spectra, such as the effect of doping, the presence of the counterions, the effect of the $\pi-\pi$ stacking of the backbones, the effect of the side chains, and the effect of the bending and twisting of the chains. In addition, we attempt to provide a clear guideline for the use of correct language for the assignment of polaron, bipolaron, and polaron pair, as they apply to the electronic state and optical spectra of ionized $\pi$ systems. We also point out that, despite the fact that the terminology of polaron and bipolaron (as well as polaron pairs) has been discussed in the literature accurately for several decades, the descriptions using the earlier models and inaccurate assignment of spectral features based upon these models continue to be widely used, propagating misconception and confusion. We hope that this paper will serve to provide additional clarity to these issues and encourage the organic electronic community working on doping to accept and adopt the more modern and physically correct picture of the states and the transition that occur upon doping.

\section{METHODS}

II.I. Experimental Methods. II.I.I. Materials. P3HT was purchased from Ossila Ltd. (number-average molecular weight $M_{\mathrm{n}} \sim 29 \mathrm{~kg} \mathrm{~mol}{ }^{-1}$; polydispersity index (PDI) $\sim 2.2$; regioregularity $\sim 96 \%)$, and $\mathrm{P}\left(\mathrm{g}_{4} 2 \mathrm{~T}-\mathrm{T}\right)\left(M_{\mathrm{n}} \sim 24 \mathrm{~kg} \mathrm{~mol}^{-1}\right.$; PDI $\sim 3.3)$ was synthesized according to ref 15 . Mo(tfd$\left.\mathrm{COCF}_{3}\right)_{3}$ was synthesized according to ref 53. The solvents chlorobenzene, $o$-dichlorobenzene, chloroform, and acetonitrile with purity $>99 \%$ were purchased from Sigma-Aldrich.

II.I.II. Sample Preparation and Doping. Thin films of $\mathrm{P} 3 \mathrm{HT}$ and $\mathrm{P}\left(\mathrm{g}_{4} 2 \mathrm{~T}-\mathrm{T}\right)$ were spin-coated onto $\mathrm{BaF}_{2}$ substrates from $1: 1 \mathrm{w} / \mathrm{w}$ chlorobenzene/o-dichlorobenzene at $60{ }^{\circ} \mathrm{C}$ and chloroform at room temperature, respectively $\left(7.5 \mathrm{~g} \mathrm{~L}^{-1}\right)$. Sequential doping with $\mathrm{Mo}\left(\mathrm{tfd}-\mathrm{COCF}_{3}\right)_{3}$ was done by dropcasting $2.5 \mathrm{~g} \mathrm{~L}^{-1}$ solutions of the dopant in acetonitrile onto polymer films and spinning off the remaining solution after 3 min.

II.I.III. UV-Vis-Near-Infrared (NIR) and Infrared Spectroscopy. Absorption measurements were performed in transmission with a PerkinElmer Lambda 900 spectrophotometer in the UV-vis-NIR region and a PerkinElmer FTIR Spectrometer "Frontier" in the infrared region. The resulting spectra were stitched together.

II.II. Theoretical Methods. During the simulations, we used two methods: single oligomers, without and with counterions, were simulated using DFT and time-dependent DFT (TD-DFT). Larger systems, including stacked polymer chains, as well as oligomers with side chains during temperature-induced motion, were simulated using tightbinding density functional theory (DFTB) and TD-DFTB.

II.II.I. Density Functional Theory Simulations. Polymer chains with counterions were simulated with DFT, implemented in the Gaussian 16 software package. ${ }^{54}$ For the calculation of the optical transitions (vertical excitations), we utilized the TD-DFT approach. The DFT calculations in the present study were performed using two different popular functionals, the global hybrid exchange-correlation functional B3LYP $\mathrm{P}^{55,56}$ and the range-separated hybrid functional $\omega \mathrm{B} 97 \mathrm{XD} .^{57} \mathrm{~A}$ comparison of these two functionals for the present system is discussed in Section S1 (Figures S1 and S2) in the Supporting Information. While we find that both functionals provide a similar description of the systems, B3LYP gives a better agreement with the experimental data. Hence, all of the DFT calculations presented in the main text correspond to the functional B3LYP. As a basis set, we chose 6-31G(d). This basis set proves itself as a reasonable balance between accuracy and cost-efficiency in simulations of electronic properties and UV-vis absorption spectra. ${ }^{41}$ Diffuse functions were omitted without the loss of accuracy for both self-charged and counterion-charged oligomers (see Figure S3). Selfconsistent calculations are performed in 50 iterations steps (see Figure S4 for details). The dispersion correction is not included as its effect on the absorption spectra for the case of a single oligomer with counterions is negligible (see Figure S5). For the visualization of the absorption spectra, the oscillator strengths were fitted by a Lorentzian with half-width at halfmaximum, hwhm $=0.15 \mathrm{eV}$.

II.II.II. Density Functional Tight-Binding Simulations. Density functional tight binding ${ }^{58}$ allows the simulation of larger systems compared to DFT. To describe the effects of $\pi-\pi$ stacking and temperature-induced motion of oligomers with long side chains on the absorption spectra of P3HT, we performed molecular dynamics (MD) DFTB simulations. Studied systems consisted of a single oligomer, two $\pi-\pi$ stacked oligomers, and three $\pi-\pi$-stacked oligomers without any explicit or implicit solvents. The initial optimization of the oligomer geometry was performed with the DFT. Therefore, the molecular dynamics simulations were performed without initial equilibration. Atomic motions were calculated with velocity Verlet dynamics under the Nose-Hoover thermostat with a targeted temperature of $300 \mathrm{~K}$, a time step of $1 \mathrm{fs}$, and a coupling strength of $1500 \mathrm{~cm}^{-1}$. Fermi electron filling at a temperature of $300 \mathrm{~K}$ was used to speed up convergence, and the adaptive filling was not considered during MD. A selfconsistent charge procedure was performed with tolerance $1 \times$ $10^{-5}$. Individual oligomers were simulated for up to $20 \mathrm{ps}$. Crystallites consisting of two or three stacked oligomers were simulated for 1 ps. The DFTB studies were performed for isolated polymer chains, without periodic boundary conditions. Calculations were conducted with the DFTB+ software package $^{58}$ on the SCC-DFTB3 level. ${ }^{59}$ 3ob-3-1 parameter sets ${ }^{60-62}$ were utilized during all simulations, as well as the D3 dispersion correction ${ }^{63}$ with the Becke-Johnson dumping. ${ }^{62,64}$ After obtaining geometries of the polymer chains, UV-to-IR absorption spectra were calculated with the time-dependent density functional tight-binding (TD-DFTB) method. ${ }^{65}$

II.II.III. Model for Polymer Chains. Both P3HT and P(g $\mathrm{g}_{4} 2 \mathrm{~T}$ $\mathrm{T}$ ) have rather long polymer chains, where the number of thiophene rings in the chains is ca. 100 or more. ${ }^{15}$ In our calculation, we choose the number of thiophene rings $N=18$ $(\approx 6.9 \mathrm{~nm})$ for the DFT calculations of oligomers and $N=12$ for the DFTB calculations of $\pi-\pi$-stacked chains and chains with long side chains. This is justified because the calculated absorption is practically unchanged when the oligomer length increases above $N \approx 12$ (see Figure S6). It should also be noted that much shorter chain lengths in calculations are justified because the coherence length is not expected to extend over the entire chain but rather over the distance comparable to the persistence length, which, for the system at hand, is around $3 \mathrm{~nm} .^{66-68}$

$\mathrm{P} 3 \mathrm{HT}$ and $\mathrm{P}\left(\mathrm{g}_{4} 2 \mathrm{~T}-\mathrm{T}\right)$ oligomers consist of thiophene rings (backbone) decorated with long side chains. Including the side chains explicitly into the DFT calculations represents a 
formidable task in terms of the computational time. At the same time, electronic and optical properties of P3HT and $\mathrm{P}\left(\mathrm{g}_{4} 2 \mathrm{~T}-\mathrm{T}\right)$ are primarily determined by the $\pi$-electrons residing on the carbon and sulfur atoms in the polymer backbone and are much less affected by the side chains. We performed calculations for oligomers where we subsequently cut the side chain from the full length to no side chains at all (see Figure S7). We find that the side chains with only two carbon atoms give practically the same absorption spectra as the full structures. Hence, in all DFT calculations of the oligomers reported in this paper, the side chains in $\mathrm{P} 3 \mathrm{HT}$ and $\mathrm{P}\left(\mathrm{g}_{4}\right.$ 2T-T $)$ are represented as $\mathrm{CH}_{2} \mathrm{CH}_{3}$ and $\mathrm{OCH}_{3}$ groups, respectively. The DFT calculations of PT without side chains were performed in implicit dichloromethane solvent with the CPCM model ${ }^{69}$ with a default set of parameters in Gaussian 16 . The van der Waals radii of the atoms were chosen according to the universal force field. Cavities were simulated as scaled van der Waals surfaces with scaling parameter 1.1.

Charged oligomers can be in several different spin configurations. For instance, for the total charge $Q=+2$, the oligomer can be in two configurations, singlet $(S=0)$ or triplet $(S=1)$. We calculated and compared the ground-state energies of all possible spin configurations for charged oligomers in the range from neutral polymer $Q=0$ to +4 . All polymer geometries were optimized. For singlet states, we used restricted (closed-shell) spin calculations. For all other cases, we performed unrestricted (open-shell) calculations. A similar approach to the calculation of the ground-state energy for different spin configurations has previously been used for $\mathrm{p}$ type $^{41}$ and n-type conjugated polymers ${ }^{70}$ and n-type/p-type polymer blends. ${ }^{46}$

II.II.IV. Simulation of Dopants (Counterions). Charging of $\mathrm{P} 3 \mathrm{HT}$ and $\mathrm{P}\left(\mathrm{g}_{4} 2 \mathrm{~T}-\mathrm{T}\right)$ was conducted in two ways: with the addition of positive charges to the chain without counterions and with the addition of charges and further balancing of the positively charged polymer chains by negatively charged counterions. Counterions are initially placed at an average distance of $4 \AA$ from the thiophene rings and at least $8 \AA$ apart from neighboring counterions, as was suggested by molecular dynamics studies of related systems. ${ }^{71,72}$ The geometry of $\mathrm{Cl}_{3}{ }^{-}$ was obtained after the DFT optimization. More details concerning the placement of the counterions are given in the captions of Figure S8. During geometrical optimization of the chains, the ion positions were frozen. This was done because a single oligomer represents only a small part of a realistic thin film. Positions of counterions in the film are affected by all oligomers around, which are not present in the current model. From this point of view, performing a full geometry optimization of an individual oligomer with nonfrozen counterions would completely disregard the effect of the surroundings. Note that a similar placement of counterions was recently used for DFT calculations of the intrinsic capacitance of conducting polymers. ${ }^{73}$ For each oxidation level, five different counterion positions were calculated, as shown in Figure S8.

In the experimental films, $\mathrm{Mo}\left(\mathrm{tfd}-\mathrm{COCF}_{3}\right)_{3}$ complexes were used as dopants and thus the counterions are $\mathrm{Mo}(\mathrm{tfd}-$ $\left.\mathrm{COCF}_{3}\right)_{3}{ }^{-}$. These are large molecules, and their utilization in the calculations requires significant computational efforts. Hence, in the calculations, we instead used ions $\mathrm{Cl}_{3}{ }^{-}$that are much smaller. Note that in most cases (an exception has been recently reported ${ }^{74}$ ) the orbitals on counterions do not appreciably mix with those of polymer chains nor their absorption spectra overlap with those of the polymers. This is illustrated in Figure $\mathrm{S} 9$ showing the absorption spectra of $\mathrm{Cl}_{3}{ }^{-}$. The main effect of the counterions is that they give rise to an electrostatic potential that affects localization of charge carriers in the polymeric chains. Note also that the calculated positions of the absorption peaks strongly depend on the choice of the functional (see Section S1), and therefore, DFT-based predictions should be considered to be of semiquantitative character. Hence, the exact type of counterions is not expected to be important for the electronic and optical properties of oligomers, and our choice of counterions is motivated by the computational efficiency. Note that we also tried other counterions often used in conductive polymers, including $\mathrm{Cl}^{-}, \mathrm{NO}_{3}{ }^{-}$, tosylate, and $\mathrm{F}_{4} \mathrm{TCNQ}{ }^{-}$(2,3,5,6-tetrafluoro7,7,8,8-tetracyanoquinodimethane). We find that for some of these counterions $\left(\mathrm{NO}_{3}{ }^{-}\right.$, tosylate), the self-interaction errors of DFT are too high (leading to the charge $Q \approx-0.5$ to -0.85 $e$ instead of the expected $-1 e)$. Note that the delocalization errors caused by incomplete self-interaction corrections represent one of the known issues of the DFT leading to the inaccuracies in the description of the charge transfer. ${ }^{75,76}$ Also, the calculations $\mathrm{Cl}^{-}$and $\mathrm{F}_{4} \mathrm{TCNQ}{ }^{-}$were plagued by convergency problems. On the other hand, $\mathrm{Cl}_{3}{ }^{-}$demonstrated a good convergence and an acceptable level of self-interaction error, typically leading to the charge on the counterions $Q \approx$ $-0.95 e$. An average charge on each of the counterions was carefully controlled during the geometrical optimization. If the absolute value of the counterion charge was lower than $90 \%$ of the elementary charge, the simulation was discarded as unsuccessful.

\section{RESULTS AND DISCUSSION}

Due to the complexity of the system, there are many factors affecting the electronic structure and UV-vis and IR absorption spectra of $\mathrm{P} 3 \mathrm{HT}$ and $\mathrm{P}\left(\mathrm{g}_{4} 2 \mathrm{~T}-\mathrm{T}\right)$ thin films. We first emphasize the differences in traditional and DFT pictures of the origin of the optical transitions of oxidized polythiophenes and define the terms that we will use in the following sections. Next, we examine a small-molecule oligomeric model system in more detail. The impact of counterions on the doped polymers is then investigated using DFT and TD-DFT simulations. As a next step, DFTB-based molecular dynamics simulations and TD-DFTB simulations of $\mathrm{P} 3 \mathrm{HT}$ are conducted to investigate the influence of torsional deformation of backbones and the side-chain orientation. Further, the impact of $\pi-\pi$ stacking is studied using TDDFTB. Finally, experimental data are analyzed based on the calculated spectra.

III.I. Differences in Traditional and DFT Descriptions. The term "polaron" is defined here to indicate a singly charged $(Q=+1)$ charge carrier that has doublet spin multiplicity. In the literature, one can find different definitions of a bipolaron (see, e.g., ref 41); however, here we follow refs 34, 77, and 78 in defining a bipolaron as a doubly charged $(Q=+2)$ charge carrier in which two electrons of opposite spins occupy the same spin-degenerate energy level resulting in a singlet spin multiplicity. It is noteworthy that in the literature, in particular in organic chemistry, one often uses notations "radical cations" and "dications" for, respectively, polarons and bipolarons. Also, in physical and quantum chemistry literature, the notion of valence and conduction bands is widely used for the occupied and unoccupied manifolds of states in the oligomers. While the notion of the "band" is, strictly speaking, justified only for the 
"Traditional" approach

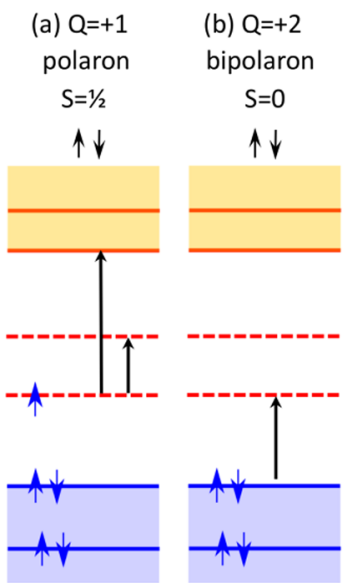

DFT approach

(d) $Q=+2$

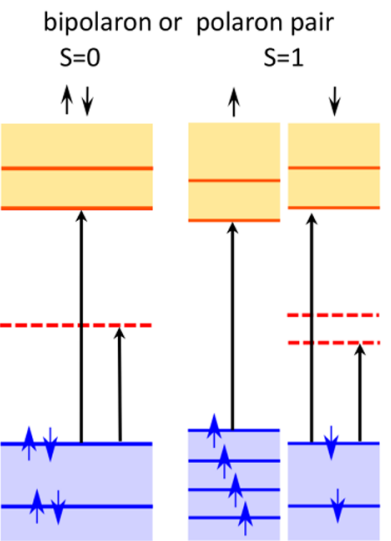

(e) Higher oxidation levels

Polarons and bipolarons

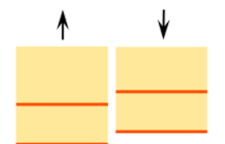

Figure 2. Electronic structure of polythiophenes according to (a, b) traditional approaches (refs 17, 18, 27, 29, and 30) and (c-e) DFT calculations ${ }^{22,32,36,41}$ for different oxidation levels ( $Q$ indicates the charge of a chain, and $S$ indicates the spin). The small blue arrows (spin-up and spin-down states) indicate occupied electronic states. The blue and red lines are occupied and unoccupied states in conduction and valence bands, respectively. (The conduction and valence bands are marked by yellow and blue background colors.) The dashed red lines indicate polaronic/ bipolaronic states in the gap. The black solid lines identify possible electronic transitions.

case on an infinite periodic structure, it de facto became common in the literature on the electronic and optical properties of conducting polymers already from the early days. $^{79}$ In the present study, we will also follow this nomenclature as it captures and well reflects the essential features of the energy structure even for oligomers of a finite length.

As discussed in Section I, p-doped polythiophenes exhibit "intermediate-" and "low-energy" absorption peaks, with the latter being more dominant at high doping levels. In the traditional model, both the polaron and bipolaron are characterized by the presence of two spin-degenerate levels in the gap between valence and conduction bands, the lowest of which is singly occupied for the polaron and empty for the bipolaron. ${ }^{17,18,27,29,30}$ The intermediate-energy peak is attributed to a combination of excitations from the half-filled "polaronic" energy levels in the gap to the conduction band and from half-filled to empty "polaronic levels" (see Figure 2a). The low-energy peak is attributed to transitions from the valence band to the empty "bipolaronic" energy levels in the gap, as shown in Figure 2b. In other words, the intermediateenergy peak is attributed solely to a doublet of singly charged carriers, i.e., polarons, whereas the low-energy peak is attributed solely to singlet dicationic species, i.e., bipolarons; accordingly, these two peaks are often referred to as polaronic and bipolaronic peaks, respectively. ${ }^{20}$

The schematic diagram of the electronic structure and optical transitions at different oxidation levels according to DFT predictions $\mathrm{s}^{22,32,36,41}$ is outlined in Figure $2 \mathrm{c}-\mathrm{e}$. For the case of a polaron $(Q=+1, S=1 / 2)$, the spin degeneracy is lifted, and there is a single empty level (i.e., empty spin orbital) in the gap; this should be contrasted with the occupation of two spin-degenerate levels by a single electron in the "early" approach (cf. Figure 2a,c). For a bipolaron $(Q=+2, S=0)$, there is a single spin-degenerate orbital in the gap, also differing from the traditional picture, where there are two such levels. We also consider the possibility of two polarons interacting to give a triplet doubly charged charge carrier (i.e., $Q=+2, S=1)$ and term these polaron pairs; for these charge carriers, there are two empty spin orbitals in the gap. For higher oxidation levels, charge carriers are polarons and/or bipolarons and are also associated with empty levels in the gap (see Figure 2e). The TD-DFT-based interpretation of the absorption peaks also differs qualitatively from the traditional approaches described above. Namely, the intermediate-energy peak ("polaronic peak") is assigned to excitation between the valence and conduction bands or a superposition of interband and valence-band-to-empty-level excitation; in contrast to the traditional assignment, it is present not only for polarons $(Q=$ $+1)$ but also for bipolarons and polaron pairs $(Q=+2)$. The low-energy peak is attributed to excitation from the valence band to the empty polaron/bipolaron (spin)orbital(s) in the gap; again in contrast to the traditional assignment, this transition is also present for all oxidation levels (i.e., polarons $(Q=+1)$, bipolarons/polaron pair $(Q=+2)$, etc. $){ }^{41}$

III.II. UV-to-IR Absorption in a Reference System: PT N $=6$ Oligomers. A quantitative agreement between theory and experiment is a prerequisite for the validation of the theoretical approach. However, because of the complexity of the present system of $\mathrm{P} 3 \mathrm{HT}$ and $\mathrm{P}\left(\mathrm{g}_{4} 2 \mathrm{~T}-\mathrm{T}\right)$ films, a possible discrepancy between theory and experiment may reflect shortcomings of the DFT-based approach utilized and/or can be related to a number of factors not included in the model, such as inhomogeneity of thin film doping, chain bending, effects of dielectric environment and water, presence of adsorbed atoms, short-chain segments, and surface effects. Therefore, to validate the method and demonstrate its predictive power, we first focus on a well-defined reference system, in which such experimental uncertainties should not be present. We chose to model a diluted system of polythiophene (PT) oligomers of fixed, well-controlled lengths and oxidation levels whose absorption spectra were studied by van Haare et al. ${ }^{17}$

The calculated electronic structure and the absorption spectra (superimposed on the experimental spectra from ref 17) of PT oligomers consisting of $N=6$ thiophene rings with the oxidation levels $Q=+1$ and +2 are shown in Figure 3. The ground state for $Q=+1$ is a polaron ( $\operatorname{spin} S=1 / 2$ ) and that for $Q=+2$ is a bipolaron $(\operatorname{spin} S=0)$. Note that for $N=6$ the 
(a) $Q=+1$, polaron
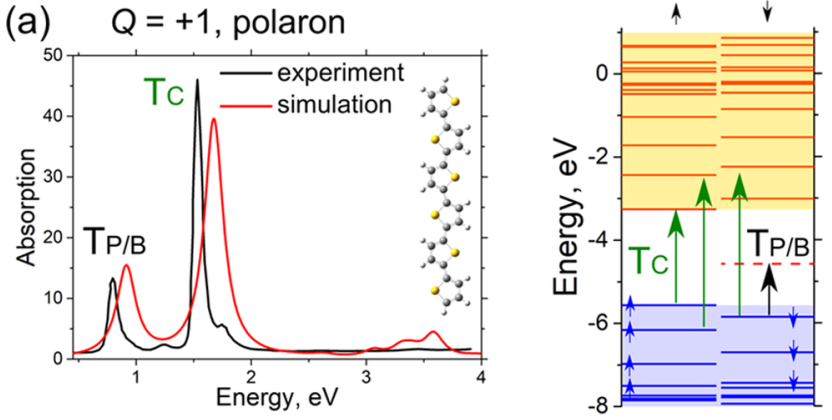

(b) $Q=+2$, bipolaron

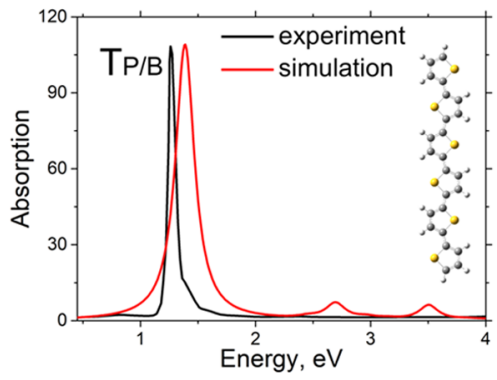

provides a validation of the utilized theoretical approach based on DFT and TD-DFT calculations.

III.III. Effects of Counterions on the Electronic Structure and Optical Absorption of P3HT and P$\left(\mathbf{g}_{4} \mathbf{2 T}-\mathrm{T}\right)$ Oligomers. III.III.I. Electronic Structure. We start our discussion with a reference case of $N=18$ oligomers without counterions. In this subsection, we present results for P3HT only because $\mathrm{P}\left(\mathrm{g}_{4} 2 \mathrm{~T}-\mathrm{T}\right)$ exhibits practically the same features. Figure 4a shows the ground-state spin multiplicity calculated for each oxidation level $(Q)$, while Figure $4 \mathrm{~b}$ shows the corresponding energy diagrams. For the polaron $(Q=+1)$, the spin degeneracy is lifted and the ground state is a doublet. We stress that in the DFT approach the spin of the polaron (as well as of all other $S>1$ charge carriers) originates from the imbalance between the number of up and down spin electrons in the valence band and not from the empty levels in the gap (which cannot have spins because they correspond to the absence of electrons). The ground state for the case $Q=+2$ is a triplet and thus corresponds to a polaron pair. The bipolaron (i.e., the singlet state with $Q=+2$ ) is energetically higher than the polaron pair. The $Q=+3$ and +4 systems are, respectively, doublet and triplet, and, therefore, they do not have any bipolaron levels (as defined above, a bipolaron is a spindegenerate charge carrier where two electrons of opposite spins occupy the same spin-degenerate energy level). It should be stressed that the spin multiplicity of the ground state and the structure of the energy levels of P3HT shown in Figure $4 \mathrm{a}, \mathrm{b}$ are the same as those for PEDOT oligomers with the same number of repeat units $(N=18) .^{41}$ This is not surprising because PEDOT is also composed of thiophene-type rings accommodating $\pi$-electrons, primarily responsible for the electronic structure and the absorption spectrum.

Let us now discuss the effect of counterions. For each of the charged oligomers $(Q=+1,+2,+3,+4)$, we calculated the electronic structure for five representative counterion positions relative to the oligomer (see Figure S8 depicting these spatial realizations). For each of these spatial realizations, we calculated energies of the ground states for different spin multiplicities $M=2 S+1$. The red dots in Figure $4 \mathrm{c}$ show the spins of the ground state with the lowest energy for each of these configurations. A comparison with the case without counterions (cf. Figure 4a) shows that in most cases the spin multiplicity remains the same, i.e., doublets for $Q=+1$ and +3 and triplets for $Q=+2$ and +4 . The only exceptions were one counterion position for $Q=+2$ and one for $Q=+4$, which both resulted in the singlet ground states (see Figure $4 \mathrm{~h}, \mathrm{i}$ and related discussion in the text below). The calculations predict a localization of polarons and bipolarons in the vicinity of counterions with localization length extending over several monomer units, as illustrated in Figure $4 \mathrm{e}-\mathrm{i}$, which shows the change in the bond length alteration as compared to the neutral oligomers. (Note that the bond length alteration changes from the aromatic to the quinoid character in the oligomer backbone where the charge is localized ${ }^{41}$ ). It is also noteworthy that in the absence of counterions, B3LYP calculations do not predict polaron localization, as illustrated in Figure 4e.

Figure $4 \mathrm{~d}$ shows the spin-resolved orbitals for different oxidation levels of the oligomers with counterions. (For each oxidation level, we chose a representative counterion position corresponding to a typical ground-state multiplicity as indicated in the figure.) As expected, the positions of the individual energy levels are affected by the presence of 
(a)
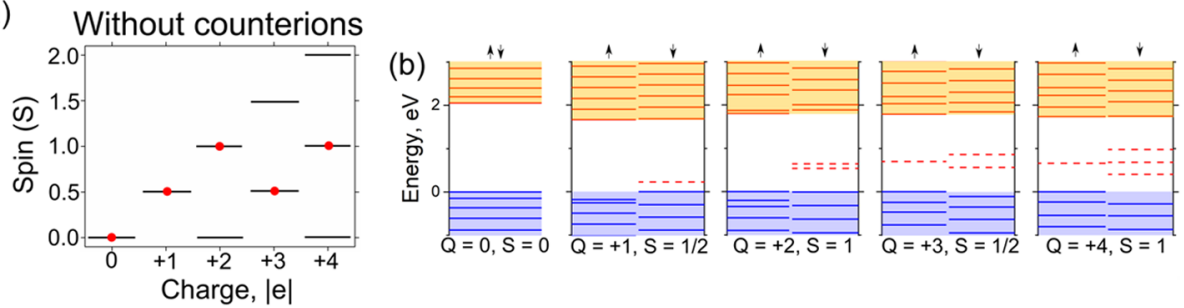

(c)
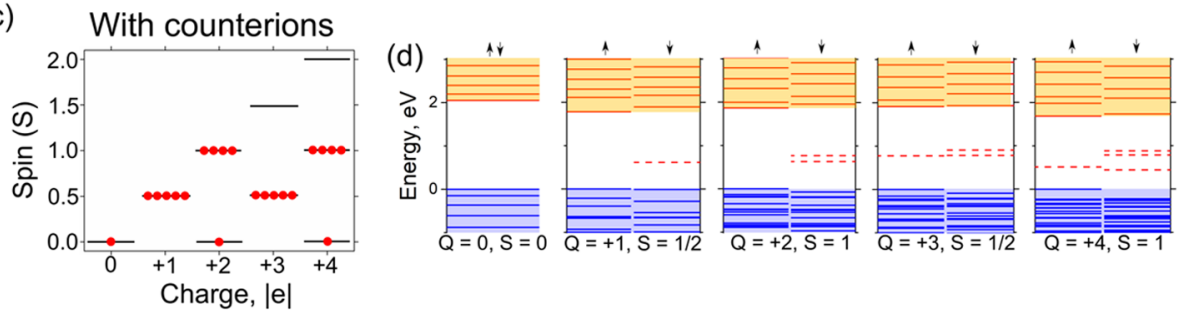

One charge in a chain $(Q=+1)$

(e) Without counterions

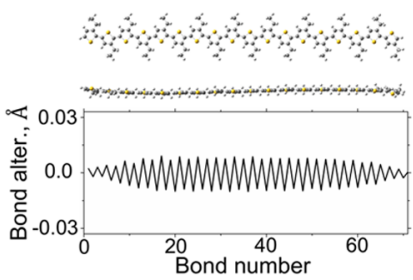

(f) realization 1
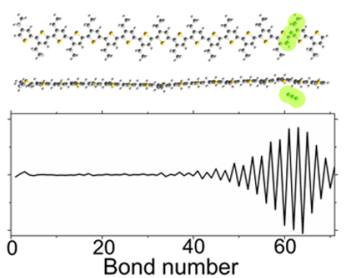

(g) realization 2

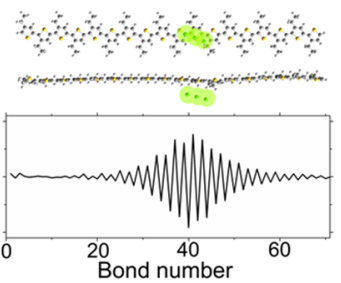

Two charges in a chain $(Q=+2)$

(h) realization 1 , bipolaron (singlet)

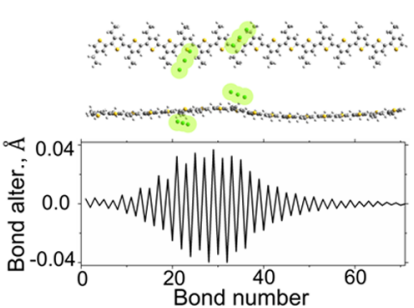

(i) realization 2, polaron pair (triplet)
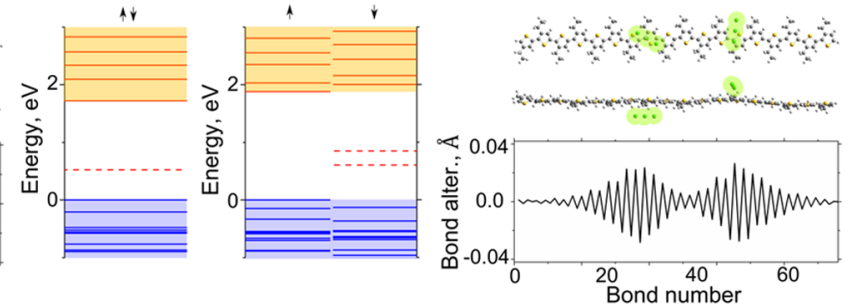

Figure 4. Total electron spin of the lowest-energy ground states of the P3HT oligomers without and with counterions ((a) and (c), respectively). For the case of oligomers with counterions, five different realizations (shown in Figure S8) were calculated; the red dots in (c) indicate the spin of the ground state with the lowest energy for each of these configurations. (b, d) Energy diagram for P3HT oligomers at different oxidation levels for the cases without and with counterions, respectively. For the case with counterions, the energy diagrams are chosen for representative realizations where the ground energy spin state is the same as for the case without counterions, namely, realization $1,1,1,2$ for the cases $Q=+1,+2,+3,+4$ in Figure S8. The blue and red lines are occupied and unoccupied energy levels. The dashed red lines represent (unoccupied) polaronic states in the gap. The yellow and blue backgrounds mark the conduction and valence bands, respectively. $(\mathrm{e}-\mathrm{g})$ Bond length alteration for the case of $Q=+1$ without counterions and for different positions of counterions, as illustrated in the upper panel; the counterions are highlighted in green. The bond numbers and the bond length alteration are defined in Figure S10. Examples of two different counterion configurations for the case $Q=+2$ leading to the formation of $(\mathrm{h})$ a bipolaron and (i) a polaron pair. The corresponding energy diagram and the bond length alterations are shown. All calculations $(\mathrm{a}-\mathrm{i})$ correspond to the number of thiophene units $N=18$. In all energy diagrams, the highest occupied (spin-resolved) orbital levels of the valence bands are aligned at $E=0$.

counterions, ${ }^{73,80,81}$ but the overall energetic structure remains similar to that without counterions.

Figure $4 \mathrm{~h}, \mathrm{i}$ illustrates the cases for which two different counterion positions lead to different ground-state spin multiplicities, singlet and triplet, corresponding to, respectively, a bipolaron and a polaron pair. Bipolaron, rather than polaron pair, ground states typically arise when two counterions are relatively close to each other, which helps to overcome Coulombic repulsion between two holes. Note that bipolarons can coexist with polarons on the same oligomers. This is the case for $Q=+3$ in Figure $4 d$, for which there are three empty spin orbitals in the gap. The lowest of these are spindegenerate with spin-up and spin-down having the same energy levels and identical orbitals, which correspond to the bipolaron, whereas the remaining empty spin-down spin orbital in the gap is associated with the polaron. Note that the coexistence of polarons and bipolarons on the same polymer chain was also predicted for n-doped polymers, such as the ladder polymer poly(benzimidazobenzophenanthroline) $(\mathrm{BBL}) .^{70}$ The coexistence of multiple polarons on a single 
without counterions

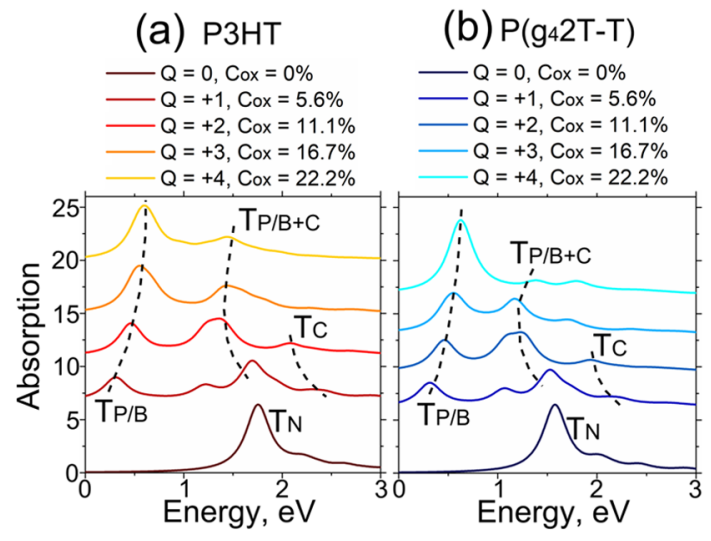

(e)

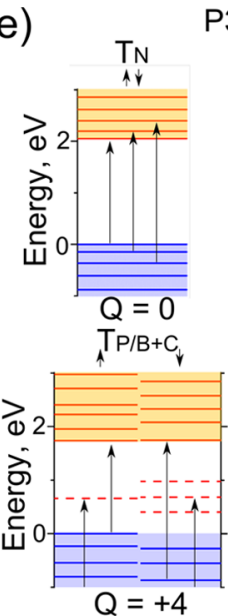

P3HT
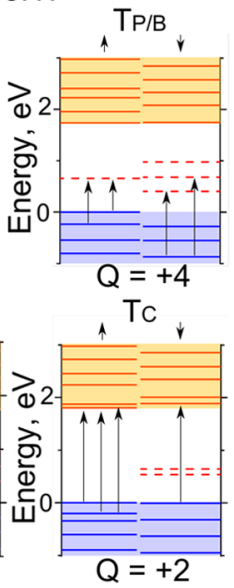

(c)

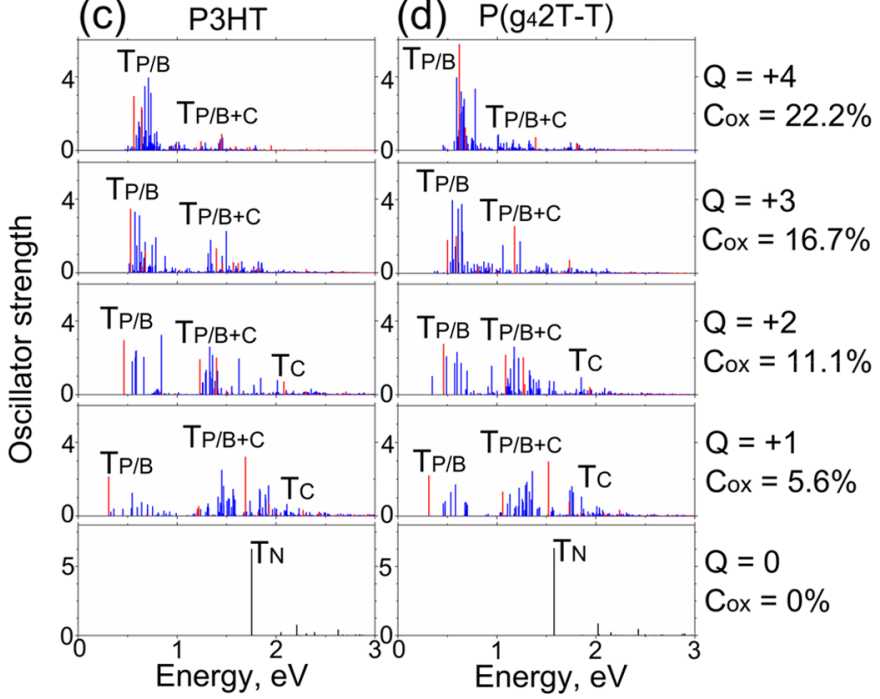

P3HT

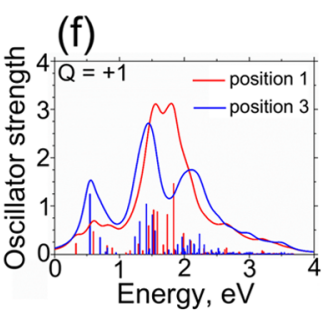

(g)

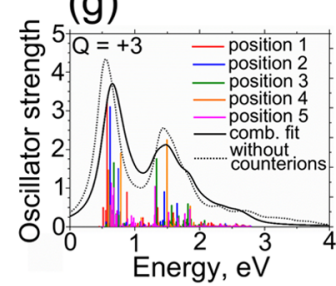

Figure 5. Evolution of the absorption spectra of (a) P3HT and (b) $\mathrm{P}\left(\mathrm{g}_{4} 2 \mathrm{~T}-\mathrm{T}\right)$ with the oxidation level for the case without counterions. The transition from dark to bright colors corresponds to the increase in the oxidation level. Comparison of the absorption spectra of (c) P3HT and (d) $\mathrm{P}\left(\mathrm{g}_{4} 2 \mathrm{~T}-\mathrm{T}\right)$ for different oxidation levels for the cases with (blue lines) and without (red lines) counterions. For the sake of clarity, only the oscillator strengths are shown (without the Lorentzian fit). (e) Illustration of the electronic transitions contributing to the main peaks in the absorption spectra of the oxidized polymers, $\mathrm{T}_{\mathrm{P} / \mathrm{B}}, \mathrm{T}_{\mathrm{P} / \mathrm{B}+\mathrm{C}}$, and $\mathrm{T}_{\mathrm{C}}$. The peak $\mathrm{T}_{\mathrm{N}}$ corresponds to the absorption spectrum of a neutral polymer chain. (f) Absorption spectra of the P3HT oligomers for two different counterion realizations for $Q=+1$-in position 1 , the counterion is located near the oligomer side, while in position 3 the counterion is above its middle thiophene units (the exact geometry can be found in Figure S8). (g) Absorption spectra of the P3HT oligomers for five different counterion realizations for $Q=+3$ and their combined averaged spectrum (solid line). Panels (f) and (g) show both oscillatory strength and their Lorentzian fit.

chain was experimentally shown for the case of poly(3decylthiophene). ${ }^{23}$

III.III.II. UV-to-IR Absorption Spectra. Figure 5 shows the simulated absorption spectra of $\mathrm{P} 3 \mathrm{HT}$ and $\mathrm{P}\left(\mathrm{g}_{4} 2 \mathrm{~T}-\mathrm{T}\right)$ without and with counterions for different oxidation levels: $C_{\mathrm{ox}}=5.5$, $11.1,16.6$, and $22.2 \%$. (The oxidation level is defined as the number of charges per thiophene ring.) These values correspond to the charge $Q=+1,+2,+3$, and +4 per chain.

Let us first discuss the spectra for the system without the counterions (Figure $5 \mathrm{a}-\mathrm{d}$ ). The spectra of charged polymers show absorption peaks that can be divided into three main groups, as outlined in Figure 5e. The peaks $\mathrm{T}_{\mathrm{P} / \mathrm{B}}$ and $\mathrm{T}_{\mathrm{C}}$ have the same origin as discussed in Section III.I for polythiophenes in general and in Section III.II for an oligothiophene, i.e., they correspond to the transitions from the valence band to the empty polaronic/bipolaronic levels and from the valence band to the conduction band, respectively. A third peak, $\mathrm{T}_{\mathrm{P} / \mathrm{B}+\mathrm{C}}$, corresponds to a superposition of excitations from the valence band into polaronic/bipolaronic levels and from the valence band into the conduction band. Calculated spectra exhibit a clear pattern with increasing oxidation level, as shown in Figure 5a,b: $\mathrm{T}_{\mathrm{P} / \mathrm{B}}$ becomes stronger and shifts to higher energies, whereas $\mathrm{T}_{C}$ and $\mathrm{T}_{\mathrm{P} / \mathrm{B}+\mathrm{C}}$ become progressively weaker, with $\mathrm{T}_{\mathrm{C}}$ disappearing at higher oxidation levels (16\% and more). This behavior reflects the evolution of the electronic structure with the oxidation level (Figure $4 \mathrm{~b}$ ) where progressively more polaronic/bipolaronic states in the gap emerge. This also explains the shift of $\mathrm{T}_{\mathrm{P} / \mathrm{B}}$ to higher energies because more highlying energy polaronic/bipolaronic levels become available in the gap, as shown in Figure $4 \mathrm{~b}$. Note that for $C_{\mathrm{ox}} \approx 14 \%$ the heights of the peaks $\mathrm{T}_{\mathrm{P} / \mathrm{B}}$ and $\mathrm{T}_{\mathrm{P} / \mathrm{B}+\mathrm{C}}$ become approximately equal. This can be used to estimate the oxidation level of a polymer film from its absorption spectrum if the films are doped homogeneously. It is noteworthy that the calculated 

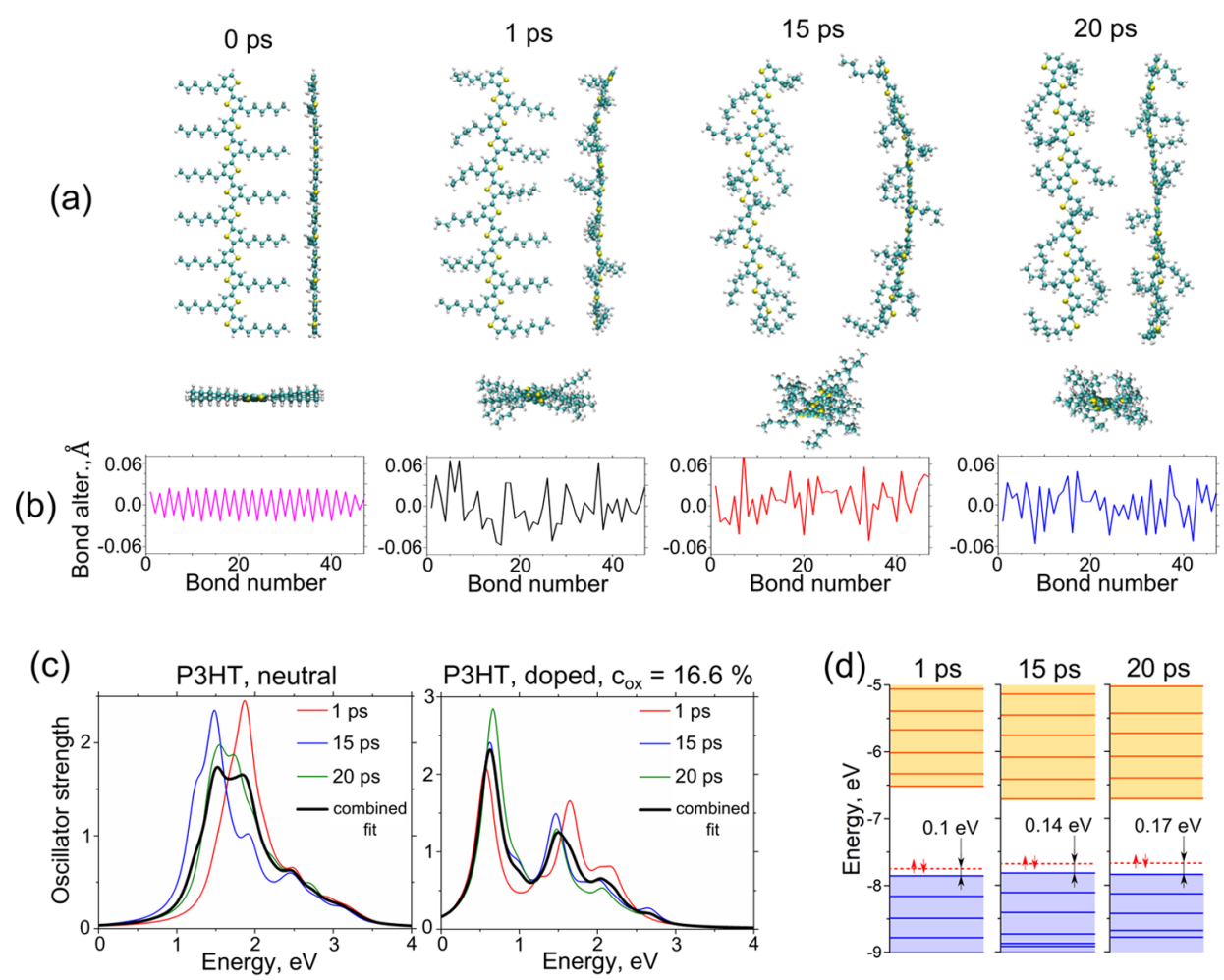

(e) Bilayer, 1 ps

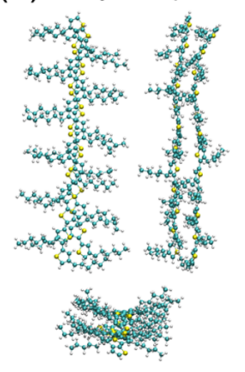

Trilayer, 1 ps

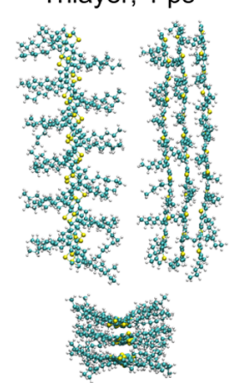

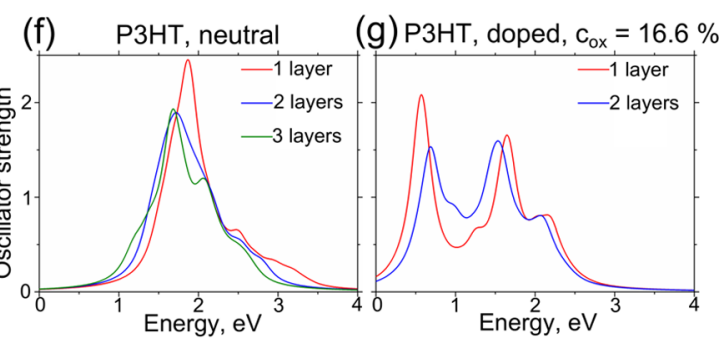

Figure 6. (a) Geometrical structure of a P3HT oligomer with $N=12(Q=+2)$ after an MD-DFTB run and (b) corresponding C $-\mathrm{C}$ backbone bond alteration at $t=0,1,15$, and 20 ps. (c) Absorption spectra of a neutral and a doped P3HT oligomer with $Q=+2\left(C_{\mathrm{ox}}=16.6 \%\right)$ at $t=1,15$, and 20 ps. The bold black line shows the averaged spectrum. (a) Band diagrams of P3HT at 1, 15, and 20 ps. (e) Geometrical structure of bi- and trilayer P3HT after a 1 ps DFTB MD run; $C_{\mathrm{ox}}=16.6 \%(\mathrm{Q}=+2)$. The absorption spectra of neutral $(\mathrm{f})$ and doped $(\mathrm{g})$ P3HT multilayers with $C_{\mathrm{ox}}=$ $16.6 \%$ at $t=1,15$, and 20 ps. The bond numbers and the bond length alteration are defined in Figure S10.

evolution of the absorption spectra is similar to that reported for PEDOT. ${ }^{41}$

Let us now discuss the influence of counterions on the absorption spectra. The positions of counterions significantly affect the electronic structure of the polymers, which, in turn, affects the absorption spectra. An example is depicted in Figure 5f, where the absorption spectra for the P3HT chains with the $C_{\mathrm{ox}} \approx 5.6 \%(Q=+1)$ are calculated for two different counterion positions. In position 1 , the counterion is located at the end of the polymer chain, whereas in position 3 , the counterion is located at the center of the chain (see Figure S8). This change in the geometry of the system results in different absorption spectra for two oligomers with the same oxidation level. Absorption peaks of $\mathrm{P} 3 \mathrm{HT}$ chains with three counterions $(Q=+3)$ for five different spatial counterion orientations are depicted in Figure $5 \mathrm{~g}$. As for the case of $Q=+1$, each arrangement of counterions leads to a different spectrum. However, averaging over these five arrangements results in two broad absorption peaks residing at the positions close to the corresponding peaks for the counterion-free chains with the same charge, as shown in Figure 5a. We performed calculations for chains with different oxidation levels and find that the same conclusion holds for all considered systems for both polymers P3HT and $\mathrm{P}\left(\mathrm{g}_{4} 2 \mathrm{~T}-\mathrm{T}\right)$ (see Figure $\left.5 \mathrm{c}, \mathrm{d}\right)$. Thus, the effect of counterions on the absorption spectra of polythiophenes with one or several charges can be summarized as follows: the presence of counterions leads to a significant broadening of the absorption peaks, whereas the positions of the peaks remain close to those without counterions.

III.IV. Absorption Spectra in Polymer Chains That Display Backbone Torsion and $\boldsymbol{\pi}-\boldsymbol{\pi}$ Stacking. $\mathrm{P}\left(\mathrm{g}_{4} 2 \mathrm{~T}-\mathrm{T}\right)$ thin films are mostly disordered with some degree of crystallinity, while regioregular P3HT films have semicrystalline character. ${ }^{25,31,48,52,82}$ The chains of both P3HT and $\mathrm{P}\left(\mathrm{g}_{4} 2 \mathrm{~T}-\mathrm{T}\right)$ are nonplanar; both the backbones and side chains are in constant temperature-induced movement, which causes twisting of the side chains and changes of the torsion angles along the backbones. In this section, we will study the effect of 
twisting and the effect of $\pi-\pi$ stacking on the absorption spectra. In the treatment of chain twisting, it is important to account for the effect of the side chains, as they strongly affect the chain movement and bending. The TD-DFT simulation of polythiophenes decorated with side chains and/or several $\pi-\pi$ stacked chains is computationally challenging due to a large size of the systems. Therefore, to study the effect of backbone torsions and $\pi-\pi$ stacking on the absorption spectra of polythiophenes, we use the TD-DFTB approach combined with DFTB-based molecular dynamics (MD) simulations, which allows us to calculate much larger systems than conventional DFT.

Figure 6a shows snapshots of the geometry of a P3HT oligomer with charge $Q=+2$ calculated using MD simulations on the DFTB level of theory during a time interval of $0-20$ ps. The initial geometry of the thiophene rings and side chains is close to planar. With time, the side chains start to move, changing the torsion angles and bond lengths in the thiophene rings. At $t=15 \mathrm{ps}$, side chains bend relative to the oligomer backbone, and at $t=20 \mathrm{ps}$, some of the side chains twist so strongly, so that they are in part located on the top and under the thiophene rings. Twisting of the backbone results in significant changes in the bond lengths, as illustrated in Figure $6 \mathrm{~b}$.

Figure $6 \mathrm{c}$ shows the absorption spectra of a neutral P3HT oligomer and the oligomer with $Q=+2$ calculated for different times $t=1,10,15$, and 20 ps. All of them show similar behavior where, however, the positions and the strengths of the absorption peaks vary for different spatial conformations of the chains. The black solid line shows the spectrum averaged over spatial conformations at different moments of time. It shows a broadening relative to a spectrum corresponding to a given confirmation. This is similar to the case of a chain with counterions discussed in Section III.III, in which the broadening was caused by averaging over different counterion positions. This similarity is not a coincidence. Indeed, Figure $6 \mathrm{~d}$ shows the electronic structure of a chain with $Q=+2$ at different times. While the structure of the energy levels is similar, the exact position of the bipolaron levels in the gap is different for different moments of time. This is related to the changes in the bond length and angles that take place during the torsional motion of the chains (see Figure $6 \mathrm{~b}$ ). The difference in the energy levels leads to different absorption spectra, in a similar way as discussed above for the case of counterions.

To analyze the effect of $\pi-\pi$ stacking on UV-vis absorption spectra, we simulated bilayer and trilayer P3HT crystallites for $t=1 \mathrm{ps}$, for both neutral and $16.6 \%$ oxidized states. From the nanostructure snapshots shown in Figure $6 \mathrm{~d}$, the $\pi-\pi$ stacking is clearly observed. Absorption spectra in Figure 6f show a broadening of the main peaks as the number of chains in the layer is increased. Unfortunately, TD-DFTB simulation of the doped trilayer sample is too computationally demanding (due to a large number of excited states), and, therefore, Figure $5 f$ shows the spectra for a bilayer only. We conclude that the effect of twisting of the polymer chains and the effect of $\pi-\pi$ coupling on the absorption spectra of polythiophenes with side chains is qualitatively similar to that of counterions and manifests itself in a broadening of the absorption peaks.

III.V. Comparison of Simulation and Experimental Studies. Figure 7 shows the measured absorption spectra for undoped $\mathrm{P} 3 \mathrm{HT}$ and $\mathrm{P}\left(\mathrm{g}_{4} 2 \mathrm{~T}-\mathrm{T}\right)$, as well as thin films doped with $\mathrm{Mo}\left(\mathrm{tfd}-\mathrm{COCF}_{3}\right)_{3}$ (see Figure 1c for chemical structure).
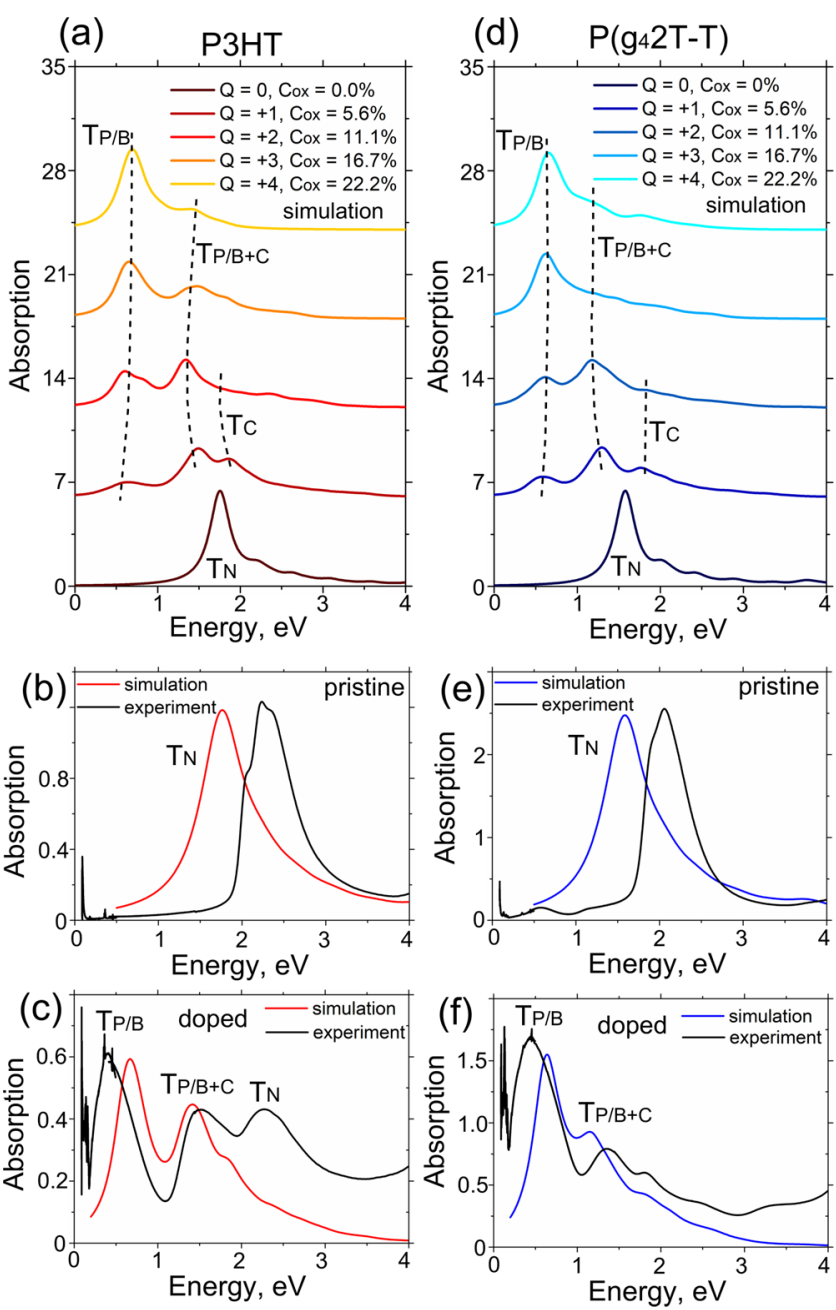

Figure 7. Evolution of the absorption spectra of (a) P3HT and (d) $\mathrm{P}\left(\mathrm{g}_{4} 2 \mathrm{~T}-\mathrm{T}\right)$ with the increase in the oxidation level for the case with counterions. (Note that absorption spectra are obtained by superposition of the Lorentzian-fitted oscillator strengths in Figure 5c,d.) The transition from dark to bright colors corresponds to the increase in the oxidation level. Comparison of experimentally obtained absorption spectra with the theoretical ones for pristine (b) P3HT and (e) $\mathrm{P}\left(\mathrm{g}_{4} 2 \mathrm{~T}-\mathrm{T}\right)$ (note that the pristine polymer is in fact slightly oxygen-doped due to its low ionization energy) and (c, f) the same polymers sequentially doped with $\mathrm{Mo}\left(\mathrm{tfd}-\mathrm{COCF}_{3}\right)_{3}$. The calculated curves include the averaged spectra for all calculated doping levels $\left(C_{\text {ox }}=5.6,11.1,6.7,22.2 \%\right)$. The black curves are experimental studies; the red and blue curves are simulation results for P3HT and $\mathrm{P}\left(\mathrm{g}_{4} 2 \mathrm{~T}-\mathrm{T}\right)$. The calculations are performed for oligomers with $N=18$ monomer units.

The calculated spectra accounting for the broadening due to the effect of counterions are shown in Figure $5 c, d$ and are also displayed in Figure 7a,d. We note that, as will be discussed below, the experimental samples are likely to contain both doped or nondoped domains. Hence, for the sake of convenience of the comparison to the experiment, each displayed theoretical spectrum in Figure 7 represents the averaged spectrum for all calculated doping levels $\left(C_{\mathrm{ox}}=5.6\right.$, $11.1,16.7,22.2 \%)$. This corresponds to the average doping level of ca. $14 \%$, close to the experimental one (see below).

Let us start with the case of P3HT. For the doped films, the spectrum shows three peaks. The comparison with the calculated spectra shows that the first two peaks match the 
corresponding theoretical peaks $\left(\mathrm{T}_{\mathrm{P} / \mathrm{B}}\right.$ and $\left.\mathrm{T}_{\mathrm{P} / \mathrm{B}+\mathrm{C}}\right)$. The lowenergy peak $\mathrm{T}_{\mathrm{P} / \mathrm{B}}$ (at ca. $0.4 \mathrm{eV}$ ) is the most pronounced, which suggests that the doping level of the sample is rather high (see Figure 7a). Although calculated spectra for high doping levels are dominated by only two peaks, $\mathrm{T}_{\mathrm{P} / \mathrm{B}}$ and $\mathrm{T}_{\mathrm{P} / \mathrm{B}+\mathrm{C}}$, the third (high-energy) experimental peak (at ca. 2.3 $\mathrm{eV}$ ) matches exactly the absorption peak of the neutral (undoped) film, excluding vibronic spectrum. This leads us to conclude that some regions remain undoped. Therefore, based on our DFT calculations, the assignment of the experimentally observed peaks for a doped P3HT is as follows. The lowenergy peak at ca. $0.4 \mathrm{eV}$ corresponds to $\mathrm{T}_{\mathrm{P} / \mathrm{B}}$, the intermediate-energy peak at ca. $1.5 \mathrm{eV}$ corresponds to

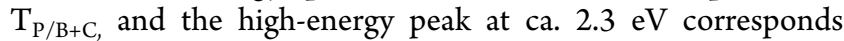
to $\mathrm{T}_{\mathrm{N}}$ due to the presence of undoped polymer segments in the film (see Figure 7a).

To estimate the oxidation level from the experimental spectra, we used a molar attenuation coefficient of $\varepsilon=(4.1 \pm$ $0.2) \times 10^{3} \mathrm{~m}^{2} \mathrm{~mol}^{-1}$ for the $\mathrm{T}_{\mathrm{P} / \mathrm{B}+\mathrm{C}}$ transition of oxidized P3HT with the peak at $1.55 \mathrm{eV}(800 \mathrm{~nm})$, which we have obtained from spectroelectrochemistry measurements. ${ }^{83}$ The Beer-Lambert law $A=N_{\mathrm{v}} \cdot \varepsilon \cdot d$ relates the peak absorbance $A$ of doped P3HT at $1.55 \mathrm{eV}$ to the molar attenuation coefficient $\varepsilon$, where $d=54 \mathrm{~nm}$ is the film thickness. We obtain a polaron density of $N_{\mathrm{v}}=(4.2 \pm 0.5) \times 10^{26} \mathrm{~m}^{-3}$, which translates to an oxidation level of $(11 \pm 1) \%$ per thiophene for $\mathrm{Mo}(\mathrm{tfd}-$ $\left.\mathrm{COCF}_{3}\right)_{3}$-doped P3HT (assuming a density of $1.1 \mathrm{~g} \mathrm{~cm}^{-3}$ ).

Let us now proceed to the $\mathrm{P}\left(\mathrm{g}_{4} 2 \mathrm{~T}-\mathrm{T}\right)$ film. The low-energy and the intermediate-energy peaks for the doped sample are very close to the corresponding peaks for P3HT (0.4 and 1.5 $\mathrm{eV}$ vs 0.4 and $1.4 \mathrm{eV}$ ). Therefore, the assignment of these peaks is the same as that for the P3HT film. In contrast to the doped P3HT, the high-energy peak at ca. $2.3 \mathrm{eV}$ is not present in the doped $\mathrm{P}\left(\mathrm{g}_{4}\right.$ 2T-T $)$ film; we, therefore, conclude that the $\mathrm{P}\left(\mathrm{g}_{4} 2 \mathrm{~T}-\mathrm{T}\right)$ film does not contain undoped regions. We estimated the oxidation level from the experimental spectra assuming that the molar attenuation coefficient of the $\mathrm{T}_{\mathrm{P} / \mathrm{B}+\mathrm{C}}$ transition of oxidized $\mathrm{P}\left(\mathrm{g}_{4} 2 \mathrm{~T}-\mathrm{T}\right)$ with the peak at $1.38 \mathrm{eV}(900$ $\mathrm{nm})$ is the same as that of oxidized P3HT. We obtain a value of $N_{\mathrm{v}}=(4.6 \pm 0.5) \times 10^{26} \mathrm{~m}^{-3}$ for a film thickness of $d=80$ $\mathrm{nm}$, which indicates an oxidation level of $(15 \pm 2) \%$ per thiophene for $\mathrm{Mo}\left(\mathrm{tfd}-\mathrm{COCF}_{3}\right)_{3}$-doped $\mathrm{P}\left(\mathrm{g}_{4} 2 \mathrm{~T}-\mathrm{T}\right)$. As discussed above, the ratio of peak heights of $\mathrm{T}_{\mathrm{P} / \mathrm{B}}$ and $\mathrm{T}_{\mathrm{P} / \mathrm{B}+\mathrm{C}}$ depends on the doping level (see Figure 5a). A comparison of the experimental peak ratio to the theoretical one is consistent with an oxidation level of ca. $16-20 \%$ for $\mathrm{P}\left(\mathrm{g}_{4} 2 \mathrm{~T}-\mathrm{T}\right)$, which is in agreement with the above estimation taking into account experimental uncertainties and systematic errors in DFT.

As already mentioned in Sections I and III.I, the polymers with a thiophene-like backbone share the same features of the absorption spectra. In particular, similar spectral features are also observed for electrochemical doping, ${ }^{19,84}$ photoinduced de-doping, ${ }^{16}$ acid doping, ${ }^{85}$ self-doping, ${ }^{86}$ and doping with different redox dopants. ${ }^{49}$ Hence, this confirms our conclusion that the position of the absorption is largely independent of the counterions.

\section{CONCLUSIONS}

The electronic absorption of polythiophenes decorated with aliphatic and oligo ethylene glycol side chains (P3HT and $\mathrm{P}\left(\mathrm{g}_{4} 2 \mathrm{~T}-\mathrm{T}\right)$, respectively) and molecularly doped with $\mathrm{Mo}$ (tfd$\left.\mathrm{COCF}_{3}\right)_{3}{ }^{-}$counterions was studied and analyzed based on DFT-, TD-DFT-, and DFTB-based molecular dynamics simulations, paying particular attention to the understanding of the effect of counterions, side chains, $\pi-\pi$ stacking, and temperature-induced bending and twisting of the chains on the absorption spectra of the systems at hand. The calculated results are used to interpret the peaks in the measured spectra, where a good agreement between the theoretical and experimental data is found, especially when using the B3LYP, rather than $\omega \mathrm{B} 97 \mathrm{XD}$, functional.

DTF calculations predict that the absorption spectra of doped polythiophenes show three distinct peaks $\mathrm{T}_{\mathrm{P} / \mathrm{B}}, \mathrm{T}_{\mathrm{C}}$ (weak), and $\mathrm{T}_{\mathrm{P} / \mathrm{B}+\mathrm{C}}$ (see Figure $5 \mathrm{a}, \mathrm{b}$ or $7 \mathrm{a}, \mathrm{d}$ ). The peaks $\mathrm{T}_{\mathrm{P} / \mathrm{B}}$ and $\mathrm{T}_{\mathrm{C}}$ correspond to the excitations from the valence band to the polaronic/bipolaronic levels and the conduction band, respectively, whereas the peak $\mathrm{T}_{\mathrm{P} / \mathrm{B}+\mathrm{C}}$ corresponds to a superposition of excitations from the valence band to both polaronic/bipolaronic levels and the conduction band. The evolution of the calculated spectra exhibits a clear pattern with the increase in the oxidation level (see Figure 5a,b): the strength of $\mathrm{T}_{\mathrm{P} / \mathrm{B}}$ increases, whereas $\mathrm{T}_{\mathrm{C}}$ and $\mathrm{T}_{\mathrm{P} / \mathrm{B}+\mathrm{C}}$ become progressively weaker, with $\mathrm{T}_{\mathrm{C}}$ disappearing at higher oxidation levels. This behavior reflects the evolution of the electronic structure with the oxidation level (Figure 4b) where progressively more polaronic/bipolaronic states in the gap emerge.

We note that, as in other DFT studies of polythiophene materials, the calculations presented here lead to electronic orbital structures for polarons and bipolarons that are fundamentally different from those in the earlier traditional approaches from the 1980s that still dominate the current literature. DFT indicates a single unoccupied spin orbital in the band gap of the polymer for a polaron, whereas in the traditional interpretation there are two spin-degenerate levels in the gap, one of which is singly occupied and is associated with the polaronic band. DFT indicates a single spindegenerate level in the gap for a bipolaron, whereas there are two such levels in the traditional model. A qualitatively different interpretation of the absorption of polythiophenes also emerges: traditionally, the two most prominent peaks are referred to as polaron (at ca. $1.5 \mathrm{eV}$ ) and "bipolaron" (at ca. $0.4 \mathrm{eV}$ ) peaks, ${ }^{20}$ but the DFT results show that both major bands are seen for both polarons and bipolarons. This suggests that the existence of polarons or bipolarons cannot be reliably inferred from experimental absorption spectra, although the doping level is indicated by the relative strength of the two major bands.

The presence of counterions leads to a significant broadening of the absorption peaks, whereas the positions of the peaks remain close to those without counterions. The effect of $\pi-\pi$ stacking and temperature-induced bending and twisting of the chains is qualitatively similar to that of dopants and also leads to peak broadening. The origin of the above broadening is analyzed based on the calculated electronic structure. At the same time, we show that the absorption spectra are practically unaffected by the side chains because the electron density of the $\pi$-orbitals is localized along the polymer backbones.

\section{ASSOCIATED CONTENT}

\section{Supporting Information}

The Supporting Information is available free of charge at https://pubs.acs.org/doi/10.1021/acs.jpcb.0c08757.

S1 evolution of the band gap and absorption spectra of P3HT with the increase of the oxidation level for the 
cases with and without counterions (dopants) calculated using functionals B3LYP and $\omega$ B97XD; S2 comparison of the experimentally obtained absorption spectra with the theoretical ones for P3HT and P(g42T-T) calculated using $\omega \mathrm{B} 97 \mathrm{XD}$; S3 absorption spectra of a charged P3HT monomer with and without diffuse functions in the basis set; S4 convergence of the absorption spectra; S5 absorption spectra of a charged P3HT monomer with and without dispersion correction; S6 dependence of the main absorption peak in the pristine P3HT with reduced side chains on oligomer length; S7 absorption spectra of P3HT and P(g42T-T) for different lengths of sidechains; S8 five different counterion positions considered for each oxidation level; S9 absorption spectra of the $\mathrm{Cl} 3$ counterion; $\mathrm{S} 10$ definition of the bond numbers (PDF)

\section{AUTHOR INFORMATION}

\section{Corresponding Author}

Igor Zozoulenko - Laboratory of Organic Electronics, Department of Science and Technology, Linköping University, 60174 Norrköping, Sweden; (1) orcid.org/0000-0002-60783006; Email: igor.zozoulenko@liu.se

\section{Authors}

Ihor Sahalianov - Laboratory of Organic Electronics, Department of Science and Technology, Linköping University, 60174 Norrköping, Sweden; (1) orcid.org/0000-0002-0609$471 \mathrm{X}$

Jonna Hynynen - Department of Chemistry and Chemical Engineering, Chalmers University of Technology, 41296 Göteborg, Sweden

Stephen Barlow - School of Chemistry and Biochemistry and Center for Organic Photonics and Electronics, Georgia Institute of Technology, Atlanta, Georgia 30332-0400, United States; (1) orcid.org/0000-0001-9059-9974

Seth R. Marder - School of Chemistry and Biochemistry and Center for Organic Photonics and Electronics, Georgia Institute of Technology, Atlanta, Georgia 30332-0400, United States; (1) orcid.org/0000-0001-6921-2536

Christian Müller - Department of Chemistry and Chemical Engineering, Chalmers University of Technology, 41296

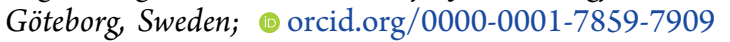

Complete contact information is available at:

https://pubs.acs.org/10.1021/acs.jpcb.0c08757

\section{Notes}

The authors declare no competing financial interest.

\section{ACKNOWLEDGMENTS}

This work was supported by the Knut and Alice Wallenberg Foundation, the Swedish Research Council (2016-05990 and 2018-03824), and the National Science Foundation (through the DMREF program, DMR-1729737). I.Z. thanks the Advanced Functional Material Center at Linköping University for support. We thank Yadong Zhang for the synthesis of $\mathrm{Mo}\left(\mathrm{tfd}-\mathrm{COCF}_{3}\right)_{3}$ and Renee Kroon for the synthesis of $\mathrm{P}\left(\mathrm{g}_{4} 2 \mathrm{~T}-\mathrm{T}\right)$. The computations were performed on resources provided by the Swedish National Infrastructure for Computing (SNIC) at NSC and HPC2N.

\section{REFERENCES}

(1) Lüssem, B.; Keum, C.-M.; Kasemann, D.; Naab, B.; Bao, Z.; Leo, K. Doped Organic Transistors. Chem. Rev. 2016, 116, 13714-13751. (2) Chew, A. R.; Ghosh, R.; Shang, Z.; Spano, F. C.; Salleo, A. Sequential Doping Reveals the Importance of Amorphous Chain Rigidity in Charge Transport of Semi-Crystalline Polymers. J. Phys. Chem. Lett. 2017, 8, 4974-4980.

(3) Kiefer, D.; et al. Double Doping of Conjugated Polymers with Monomer Molecular Dopants. Nat. Mater. 2019, 18, 149-155.

(4) Aubry, T. J.; et al. Dodecaborane-Based Dopants Designed to Shield Anion Electrostatics Lead to Increased Carrier Mobility in a Doped Conjugated Polymer. Adv. Mater. 2019, 31, No. 1805647.

(5) Moia, D.; Giovannitti, A.; Szumska, A. A.; Maria, I. P.; Rezasoltani, E.; Sachs, M.; Schnurr, M.; Barnes, P. R. F.; McCulloch, I.; Nelson, J. Design and Evaluation of Conjugated Polymers with Polar Side Chains as Electrode Materials for Electrochemical Energy Storage in Aqueous Electrolytes. Energy Environ. Sci. 2019, 12, 13491357.

(6) Flagg, L. Q.; Bischak, C. G.; Onorato, J. W.; Rashid, R. B.; Luscombe, C. K.; Ginger, D. S. Polymer Crystallinity Controls Water Uptake in Glycol Side-Chain Polymer Organic Electrochemical Transistors. J. Am. Chem. Soc. 2019, 141, 4345-4354.

(7) Cendra, C.; Giovannitti, A.; Savva, A.; Venkatraman, V.; McCulloch, I.; Salleo, A.; Inal, S.; Rivnay, J. Role of the Anion on the Transport and Structure of Organic Mixed Conductors. Adv. Funct. Mater. 2019, 29, No. 1807034.

(8) Gladisch, J.; Stavrinidou, E.; Ghosh, S.; Giovannitti, A.; Moser, M.; Zozoulenko, I.; McCulloch, I.; Berggren, M. Reversible Electronic Solid-Gel Switching of a Conjugated Polymer. Adv. Sci. 2020, 7 , No. 1901144.

(9) Veysel Tunc, A.; De Sio, A.; Riedel, D.; Deschler, F.; Da Como, E.; Parisi, J.; von Hauff, E. Molecular Doping of Low-BandgapPolymer:Fullerene Solar Cells: Effects on Transport and Solar Cells. Org. Electron. 2012, 13, 290-296.

(10) Nielsen, C. B.; McCulloch, I. Recent Advances in Transistor Performance of Polythiophenes. Prog. Polym. Sci. 2013, 38, 20532069.

(11) Giovannitti, A.; Sbircea, D.-T.; Inal, S.; Nielsen, C. B.; Bandiello, E.; Hanifi, D. A.; Sessolo, M.; Malliaras, G. G.; McCulloch, I.; Rivnay, J. Controlling the Mode of Operation of Organic Transistors through Side-Chain Engineering. Proc. Natl. Acad. Sci. U.S.A. 2016, 113, 12017.

(12) Melling, D.; Martinez, J. G.; Jager, E. W. H. Conjugated Polymer Actuators and Devices: Progress and Opportunities. Adv. Mater. 2019, 31, No. 1808210.

(13) Berggren, M.; Malliaras, G. G. How Conducting Polymer Electrodes Operate. Science 2019, 364, 233.

(14) Hynynen, J.; Kiefer, D.; Yu, L.; Kroon, R.; Munir, R.; Amassian, A.; Kemerink, M.; Müller, C. Enhanced Electrical Conductivity of Molecularly P-Doped Poly(3-Hexylthiophene) through Understanding the Correlation with Solid-State Order. Macromolecules 2017, 50, $8140-8148$.

(15) Kroon, R.; Kiefer, D.; Stegerer, D.; Yu, L.; Sommer, M.; Müller, C. Polar Side Chains Enhance Processability, Electrical Conductivity, and Thermal Stability of a Molecularly P-Doped Polythiophene. Adv. Mater. 2017, 29, No. 1700930.

(16) Wei, Q.; Mukaida, M.; Kirihara, K.; Naitoh, Y.; Ishida, T. Photoinduced Dedoping of Conducting Polymers: An Approach to Precise Control of the Carrier Concentration and Understanding Transport Properties. ACS Appl. Mater. Interfaces 2016, 8, 20542060.

(17) van Haare, J. A. E. H.; Havinga, E. E.; van Dongen, J. L. J.; Janssen, R. A. J.; Cornil, J.; Brédas, J.-L. Redox States of Long Oligothiophenes: Two Polarons on a Single Chain. Chem. - Eur. J. 1998, 4, 1509-1522.

(18) Hwang, J.; Tanner, D. B.; Schwendeman, I.; Reynolds, J. R. Optical Properties of Nondegenerate Ground-State Polymers: Three Dioxythiophene-Based Conjugated Polymers. Phys. Rev. B: Condens. Matter Mater. Phys. 2003, 67, No. 115205. 
(19) Enengl, C.; Enengl, S.; Pluczyk, S.; Havlicek, M.; Lapkowski, M.; Neugebauer, H.; Ehrenfreund, E. Doping-Induced Absorption Bands in P3ht: Polarons and Bipolarons. ChemPhysChem 2016, 17, 3836-3844.

(20) Massonnet, N.; Carella, A.; Jaudouin, O.; Rannou, P.; Laval, G.; Celle, C.; Simonato, J.-P. Improvement of the Seebeck Coefficient of Pedot:Pss by Chemical Reduction Combined with a Novel Method for Its Transfer Using Free-Standing Thin Films. J. Mater. Chem. C 2014, 2, 1278-1283.

(21) Sonmez, G. Polymeric Electrochromics. Chem. Commun. 2005, $5251-5259$.

(22) Bruno, C.; Paolucci, F.; Marcaccio, M.; Benassi, R.; Fontanesi, C.; Mucci, A.; Parenti, F.; Preti, L.; Schenetti, L.; Vanossi, D. Experimental and Theoretical Study of the P- and N-Doped States of Alkylsulfanyl Octithiophenes. J. Phys. Chem. B 2010, 114, 8585-8592.

(23) Takeda, N.; Miller, J. R. Poly(3-Decylthiophene) Radical Anions and Cations in Solution: Single and Multiple Polarons and Their Delocalization Lengths in Conjugated Polymers. J. Phys. Chem. B 2012, 116, 14715-14723.

(24) Voss, M. G.; Scholes, D. T.; Challa, J. R.; Schwartz, B. J. Ultrafast Transient Absorption Spectroscopy of Doped P3ht Films: Distinguishing Free and Trapped Polarons. Faraday Discuss. 2019, 216, 339-362.

(25) Yamamoto, J.; Furukawa, Y. Electronic and Vibrational Spectra of Positive Polarons and Bipolarons in Regioregular Poly(3Hexylthiophene) Doped with Ferric Chloride. J. Phys. Chem. B 2015, 119, 4788-4794.

(26) Koyama, T.; Nakamura, A.; Kishida, H. Microscopic Mobility of Polarons in Chemically Doped Polythiophenes Measured by Employing Photoluminescence Spectroscopy. ACS Photonics 2014, 1, 655-661.

(27) Brédas, J. L.; Wudl, F.; Heeger, A. J. Polarons and Bipolarons in Doped Polythiophene: A Theoretical Investigation. Solid State Commun. 1987, 63, 577-580.

(28) Cornil, J.; Brédas, J. L. Nature of the Optical Transitions in Charged Oligothiophenes. Adv. Mater. 1995, 7, 295-297.

(29) Stafström, S.; Brédas, J. L. Evolution of the Electronic Structure of Polyacetylene and Polythiophene as a Function of Doping Level and Lattice Conformation. Phys. Rev. B: Condens. Matter Mater. Phys. 1988, 38, 4180-4191.

(30) Cornil, J.; Beljonne, D.; Brédas, J. L. Nature of Optical Transitions in Conjugated Oligomers. Ii. Theoretical Characterization of Neutral and Doped Oligothiophenes. J. Chem. Phys. 1995, 103, 842-849.

(31) Ghosh, R.; Pochas, C. M.; Spano, F. C. Polaron Delocalization in Conjugated Polymer Films. J. Phys. Chem. C 2016, 120, 1139411406 .

(32) Heimel, G. The Optical Signature of Charges in Conjugated Polymers. ACS Central Science 2016, 2, 309-315.

(33) Dkhissi, A.; Beljonne, D.; Lazzaroni, R.; Louwet, F.; Groenendaal, L.; Brédas, J. L. Density Functional Theory and Hartree-Fock Studies of the Geometric and Electronic Structure of Neutral and Doped Ethylenedioxythiophene (Edot) Oligomers. Int. J. Quantum Chem. 2003, 91, 517-523.

(34) Zade, S. S.; Bendikov, M. Theoretical Study of Long Oligothiophene Dications: Bipolaron Vs Polaron Pair Vs Triplet State. J. Phys. Chem. B 2006, 110, 15839-15846.

(35) Salzner, U. Theoretical Investigation of Excited States of Oligothiophenes and of Their Monocations. J. Chem. Theory Comput. 2007, 3, 1143-1157.

(36) Salzner, U. Investigation of Charge Carriers in Doped Thiophene Oligomers through Theoretical Modeling of Their Uv/ Vis Spectra. J. Phys. Chem. A 2008, 112, 5458-5466.

(37) Zamoshchik, N.; Salzner, U.; Bendikov, M. Nature of Charge Carriers in Long Doped Oligothiophenes: The Effect of Counterions. J. Phys. Chem. C 2008, 112, 8408-8418.

(38) Salzner, U.; Aydin, A. Improved Prediction of Properties of ПConjugated Oligomers with Range-Separated Hybrid Density Functionals. J. Chem. Theory Comput. 2011, 7, 2568-2583.
(39) Dai, Y.; Wei, C.; Blaisten-Barojas, E. Density Functional Theory Study of Neutral and Oxidized Thiophene Oligomers. J. Chem. Phys. 2013, 139, No. 184905.

(40) Salzner, U. Electronic Structure of Conducting Organic Polymers: Insights from Time-Dependent Density Functional Theory. Wiley Interdiscip. Rev.: Comput. Mol. Sci. 2014, 4, 601-622.

(41) Zozoulenko, I.; Singh, A.; Singh, S. K.; Gueskine, V.; Crispin, X.; Berggren, M. Polarons, Bipolarons, and Absorption Spectroscopy of Pedot. ACS Appl. Polym. Mater. 2019, 1, 83-94.

(42) Farouil, L.; Alary, F.; Bedel-Pereira, E.; Heully, J.-L. Revisiting the Vibrational and Optical Properties of P3ht: A Combined Experimental and Theoretical Study. J. Phys. Chem. A 2018, 122, $6532-6545$

(43) Png, R.-Q.; Ang, M. C. Y.; Teo, M.-H.; Choo, K.-K.; Tang, C. G.; Belaineh, D.; Chua, L.-L.; Ho, P. K. H. Madelung and Hubbard Interactions in Polaron Band Model of Doped Organic Semiconductors. Nat. Commun. 2016, 7, No. 11948.

(44) Kaloni, T. P.; Schreckenbach, G.; Freund, M. S. Structural and Electronic Properties of Pristine and Doped Polythiophene: Periodic Versus Molecular Calculations. J. Phys. Chem. C 2015, 119, 39793989.

(45) Ghosh, S.; Gueskine, V.; Berggren, M.; Zozoulenko, I. V. Electronic Structures and Optical Absorption of N-Type Conducting Polymers at Different Doping Levels. J. Phys. Chem. C 2019, 123, 15467-15476.

(46) Ghosh, S.; Berggren, M.; Zozoulenko, I. Electronic Structures and Optical Properties of P-Type/N-Type Polymer Blends: Density Functional Theory Study. J. Phys. Chem. C 2020, 124, 9203-9214.

(47) Winkler, S.; Amsalem, P.; Frisch, J.; Oehzelt, M.; Heimel, G.; Koch, N. Probing the Energy Levels in Hole-Doped Molecular Semiconductors. Mater. Horiz. 2015, 2, 427-433.

(48) Kaloni, T. P.; Giesbrecht, P. K.; Schreckenbach, G.; Freund, M. S. Polythiophene: From Fundamental Perspectives to Applications. Chem. Mater. 2017, 29, 10248-10283.

(49) Heinze, J.; Frontana-Uribe, B. A.; Ludwigs, S. Electrochemistry of Conducting Polymers-Persistent Models and New Concepts. Chem. Rev. 2010, 110, 4724-4771.

(50) Ibanez, J. G.; Rincón, M. E.; Gutierrez-Granados, S.; Chahma, M.; Jaramillo-Quintero, O. A.; Frontana-Uribe, B. A. Conducting Polymers in the Fields of Energy, Environmental Remediation, and Chemical-Chiral Sensors. Chem. Rev. 2018, 118, 4731-4816.

(51) Bryan, A. M.; Santino, L. M.; Lu, Y.; Acharya, S.; D’Arcy, J. M. Conducting Polymers for Pseudocapacitive Energy Storage. Chem. Mater. 2016, 28, 5989-5998.

(52) Wang, L.; Beljonne, D. Optical Properties of Regioregular Poly(3-Hexylthiophene) Aggregates from Fully Atomistic Investigations. CrystEngComm 2016, 18, 7297-7304.

(53) Paniagua, S. A.; Baltazar, J.; Sojoudi, H.; Mohapatra, S. K.; Zhang, S.; Henderson, C. L.; Graham, S.; Barlow, S.; Marder, S. R. Production of Heavily N- and P-Doped Cvd Graphene with SolutionProcessed Redox-Active Metal-Organic Species. Mater. Horiz. 2014, $1,111-115$.

(54) Frisch, M. J. et al. Gaussian 16, revision B.01; Gaussian, Inc.: Wallingford, CT, 2016.

(55) Becke, A. D. Density-Functional Exchange-Energy Approximation with Correct Asymptotic Behavior. Phys. Rev. A 1988, 38, $3098-3100$.

(56) Lee, C.; Yang, W.; Parr, R. G. Development of the ColleSalvetti Correlation-Energy Formula into a Functional of the Electron Density. Phys. Rev. B: Condens. Matter Mater. Phys. 1988, 37, 785789.

(57) Chai, J.-D.; Head-Gordon, M. Long-Range Corrected Hybrid Density Functionals with Damped Atom-Atom Dispersion Corrections. Phys. Chem. Chem. Phys. 2008, 10, 6615-6620.

(58) Aradi, B.; Hourahine, B.; Frauenheim, T. Dftb+, a Sparse Matrix-Based Implementation of the Dftb Method. J. Phys. Chem. A 2007, 111, 5678-5684. 
(59) Gaus, M.; Cui, Q.; Elstner, M. Dftb3: Extension of the SelfConsistent-Charge Density-Functional Tight-Binding Method (SccDftb). J. Chem. Theory Comput. 2011, 7, 931-948.

(60) Gaus, M.; Goez, A.; Elstner, M. Parametrization and Benchmark of Dftb3 for Organic Molecules. J. Chem. Theory Comput. 2013, 9, 338-354.

(61) Gaus, M.; Lu, X.; Elstner, M.; Cui, Q. Parameterization of Dftb3/3ob for Sulfur and Phosphorus for Chemical and Biological Applications. J. Chem. Theory Comput. 2014, 10, 1518-1537.

(62) Kubillus, M.; Kubař, T.; Gaus, M.; Rezáč, J.; Elstner, M. Parameterization of the Dftb3 Method for $\mathrm{Br}, \mathrm{Ca}, \mathrm{Cl}, \mathrm{F}, \mathrm{I}, \mathrm{K}$, and $\mathrm{Na}$ in Organic and Biological Systems. J. Chem. Theory Comput. 2015, 11, $332-342$.

(63) Grimme, S.; Antony, J.; Ehrlich, S.; Krieg, H. A Consistent and Accurate $\mathrm{Ab}$ Initio Parametrization of Density Functional Dispersion Correction (Dft-D) for the 94 Elements H-Pu. J. Chem. Phys. 2010, 132, No. 154104.

(64) Becke, A. D.; Johnson, E. R. A Density-Functional Model of the Dispersion Interaction. J. Chem. Phys. 2005, 123, No. 154101.

(65) Niehaus, T. A.; Suhai, S.; Della Sala, F.; Lugli, P.; Elstner, M.; Seifert, G.; Frauenheim, T. Tight-Binding Approach to TimeDependent Density-Functional Response Theory. Phys. Rev. B: Condens. Matter Mater. Phys. 2001, 63, No. 085108.

(66) Heffner, G. W.; Pearson, D. S. Molecular Characterization of Poly(3-Hexylthiophene). Macromolecules 1991, 24, 6295-6299.

(67) McCulloch, B.; Ho, V.; Hoarfrost, M.; Stanley, C.; Do, C.; Heller, W. T.; Segalman, R. A. Polymer Chain Shape of Poly(3Alkylthiophenes) in Solution Using Small-Angle Neutron Scattering. Macromolecules 2013, 46, 1899-1907.

(68) Nagai, M.; Huang, J.; Zhou, T.; Huang, W. Effect of Molecular Weight on Conformational Characteristics of Poly(3-Hexyl Thiophene). J. Polym. Sci., Part B: Polym. Phys. 2017, 55, 1273-1277.

(69) Cossi, M.; Rega, N.; Scalmani, G.; Barone, V. Energies, Structures, and Electronic Properties of Molecules in Solution with the C-Pcm Solvation Model. J. Comput. Chem. 2003, 24, 669-681.

(70) Ghosh, S.; Gueskine, V.; Berggren, M.; Zozoulenko, I. V. Electronic Structures and Optical Absorption of N-Type Conducting Polymers at Different Doping Levels. J. Phys. Chem. C 2019, 123, $15467-15476$.

(71) Franco-Gonzalez, J. F.; Zozoulenko, I. V. Molecular Dynamics Study of Morphology of Doped Pedot: From Solution to Dry Phase. J. Phys. Chem. B 2017, 121, 4299-4307.

(72) Modarresi, M.; Franco-Gonzalez, J. F.; Zozoulenko, I. Computational Microscopy Study of the Granular Structure and $\mathrm{Ph}$ Dependence of Pedot:Pss. Phys. Chem. Chem. Phys. 2019, 21, 66996711.

(73) Sahalianov, I.; Singh, S. K.; Tybrandt, K.; Berggren, M.; Zozoulenko, I. The Intrinsic Volumetric Capacitance of Conducting Polymers: Pseudo-Capacitors or Double-Layer Supercapacitors? RSC Adv. 2019, 9, 42498-42508.

(74) Jacobs, I. E.; Cendra, C.; Harrelson, T. F.; Bedolla Valdez, Z. I.; Faller, R.; Salleo, A.; Moulé, A. J. Polymorphism Controls the Degree of Charge Transfer in a Molecularly Doped Semiconducting Polymer. Mater. Horiz. 2018, 5, 655-660.

(75) Cohen, A. J.; Mori-Sánchez, P.; Yang, W. Insights into Current Limitations of Density Functional Theory. Science 2008, 321, 792.

(76) Yu, H. S.; Li, S. L.; Truhlar, D. G. Perspective: Kohn-Sham Density Functional Theory Descending a Staircase. J. Chem. Phys. 2016, 145, No. 130901.

(77) Salaneck, W. R.; Friend, R. H.; Brédas, J. L. Electronic Structure of Conjugated Polymers: Consequences of Electron-Lattice Coupling. Phys. Rep. 1999, 319, 231-251.

(78) Geskin, V. M.; Brédas, J.-L. Polaron Pair Versus Bipolaron on Oligothiophene Chains: A Theoretical Study of the Singlet and Triplet States. ChemPhysChem 2003, 4, 498-505.

(79) Bredas, J. L.; Street, G. B. Polarons, Bipolarons, and Solitons in Conducting Polymers. Acc. Chem. Res. 1985, 18, 309-315.

(80) Muñoz, W. A.; Singh, S. K.; Franco-Gonzalez, J. F.; Linares, M.; Crispin, X.; Zozoulenko, I. V. Insulator to Semimetallic Transition in
Conducting Polymers. Phys. Rev. B: Condens. Matter Mater. Phys. 2016, 94, No. 205202.

(81) Munoz, W. A.; Crispin, X.; Fahlman, M.; Zozoulenko, I. V. Understanding the Impact of Film Disorder and Local Surface Potential in Ultraviolet Photoelectron Spectroscopy of Pedot. Macromol. Rapid Commun. 2018, 39, No. 1700533.

(82) Alessandri, R.; Uusitalo, J. J.; de Vries, A. H.; Havenith, R. W. A.; Marrink, S. J. Bulk Heterojunction Morphologies with Atomistic Resolution from Coarse-Grain Solvent Evaporation Simulations. J. Am. Chem. Soc. 2017, 139, 3697-3705.

(83) Untilova, V.; et al. High Thermoelectric Power Factor of Poly(3-Hexylthiophene) through in-Plane Alignment and Doping with a Molybdenum Dithiolene Complex. Macromolecules 2020, 53, 6314-6321.

(84) Bruchlos, K.; Trefz, D.; Hamidi-Sakr, A.; Brinkmann, M.; Heinze, J.; Ruff, A.; Ludwigs, S. Poly(3-Hexylthiophene) Revisited Influence of Film Deposition on the Electrochemical Behaviour and Energy Levels. Electrochim. Acta 2018, 269, 299-311.

(85) Hofmann, A. I.; Kroon, R.; Yu, L.; Müller, C. Highly Stable Doping of a Polar Polythiophene through Co-Processing with Sulfonic Acids and Bistriflimide. J. Mater. Chem. C 2018, 6, 69056910.

(86) Volkov, A. V.; Singh, S. K.; Stavrinidou, E.; Gabrielsson, R.; Franco-Gonzalez, J. F.; Cruce, A.; Chen, W. M.; Simon, D. T.; Berggren, M.; Zozoulenko, I. V. Spectroelectrochemistry and Nature of Charge Carriers in Self-Doped Conducting Polymer. Adv. Electron. Mater. 2017, 3, No. 1700096. 